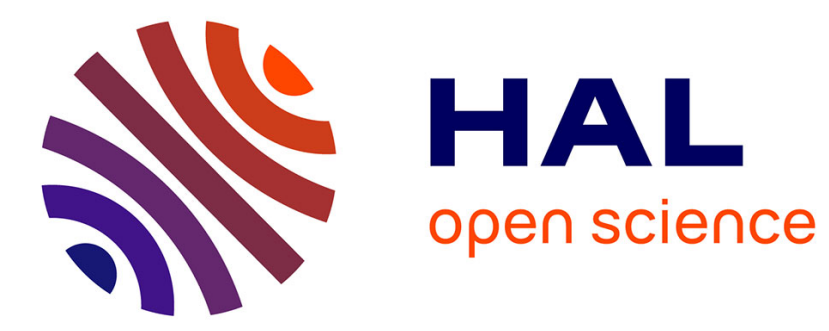

\title{
An ensemble approach to sensor fault detection and signal reconstruction for nuclear system control
}

Piero Baraldi, Antonio Cammi, Francesca Mangili, Enrico Zio

\section{To cite this version:}

Piero Baraldi, Antonio Cammi, Francesca Mangili, Enrico Zio. An ensemble approach to sensor fault detection and signal reconstruction for nuclear system control. Annals of Nuclear Energy, 2010, 37 (6), pp.778-790. 10.1016/j.anucene.2010.03.002 . hal-00609152

\section{HAL Id: hal-00609152 \\ https://hal-centralesupelec.archives-ouvertes.fr/hal-00609152}

Submitted on 25 Jul 2012

HAL is a multi-disciplinary open access archive for the deposit and dissemination of scientific research documents, whether they are published or not. The documents may come from teaching and research institutions in France or abroad, or from public or private research centers.
L'archive ouverte pluridisciplinaire HAL, est destinée au dépôt et à la diffusion de documents scientifiques de niveau recherche, publiés ou non, émanant des établissements d'enseignement et de recherche français ou étrangers, des laboratoires publics ou privés. 


\title{
An ensemble approach to sensor fault detection and signal reconstruction for nuclear system control
}

\author{
Piero Baraldi ${ }^{\mathrm{a}, 1}$, Antonio Cammi ${ }^{\mathrm{a}, 2}$, Francesca Mangili ${ }^{\mathrm{a}, 3}$ and Enrico Zio ${ }^{\mathrm{a}, 4}$ \\ ${ }^{a}$ Department of Energy, Polytechnic of Milan, via Ponzio 34/3, 20133 Milano, Italy \\ ${ }^{1}$ Corresponding author, piero.baraldi@polimi.it, Tel.: :+3902 23996355; Fax: +39 02 23996309; \\ 2antonio.cammi@polimi.it; ${ }^{3}$ francesca.mangili@mail.polimi.it; ${ }^{4}$ enrico.zio@polimi.it.
}

\begin{abstract}
To efficiently control a process, accurate sensor measurements must be provided of the signals used by the controller to decide which actions to actuate in order to maintain the system in the desired conditions. Noisy or faulty sensors must, then, be promptly detected and their signals corrected in order to avoid wrong control decisions. In this work, sensor diagnostics is tackled within an ensemble of Principal Component Analysis (PCA) models whose outcomes are aggregated by means of a local fusion (LF) strategy. The aggregated model thereby obtained is used for both the early detection and identification of faulty sensors, and for correcting their measured values. The fault detection decision logic is based on the Sequential Probability Ratio Test (SPRT). The proposed approach is demonstrated on a simulated case study concerning the pressure and level control in the pressurizer of a Pressurized Water Reactor (PWR). The obtained results show the possibility to achieve an adequate control of the process even when a sensor failure occurs.
\end{abstract}

\section{Introduction}

When a fault occurs in a sensor whose measurements are used for the control of an industrial process, a repair action must be promptly initiated since the use of incorrect information by the controller could compromise the correct functioning of the process, with potential fallbacks both on production and safety. In this context, on-line monitoring methods can provide an indication of the health of the sensors and supply an early warning of incipient faults, thus enabling to assess the reliability of the measurement and to opportunely plan the sensor maintenance. Additionally, for continuing operation while reparation is performed, the erroneous measurements should be substituted by accurate estimates of the signal true values.

The main objective of this work is to devise an on-line monitoring scheme to reduce the effects of sensor faults on the process control, by detecting the faults and by reconstructing the correct signal values. Three steps are envisaged: (a) validate the sensor measurements, (b) detect and identify faults and (c) reconstruct the correct values of the faulty signals. Steps (a) and (c) can be performed by resorting to a model that generates estimates of the correct sensors signal values based on actual readings and correlations among them; step (b) can be performed by a fault detection and identification module which determines, as early as possible, whether the sensors are behaving anomalously and identifies the faulty ones among them.

Concerning the development of a signal validation and reconstruction model, a common approach is that of using auto-associative models (Hoffmann, 2006; Holbert and Upadhyaya, 1990; Roverso et al., 2007). The practical problem, however, is that a single auto-associative model cannot handle the multiplicity of signals 
measured on a real plant (Baraldi et al., 2008; Fantoni and Mazzola, 1996; 0Fantoni et al., 2003; Zio et al., 2007). A possible way to overtake this limitation is to subdivide the signals into small overlapping groups, develop an ensemble of models, one for each group, and finally combine their outcomes. Key to building of the ensemble is the diversity of the individual models. In the approach investigated in this work, diversity is promoted by randomly generating the signals groups according to the Random Feature Selection Ensemble (RFSE) technique (Bryll et al., 2003); this is a completely random technique in which no optimization of the composition of the individual groups is sought, i.e., no relevance is given, for example, to the correlation between the signals in the groups or to their capability of reconstruction. The groups thereby created are used to develop a corresponding number of signal validation and reconstruction PCA models (Jolliffe, 2002; Diamantaras and Kung, 1996; Scholkopf et al., 1999; Moore, 1981). The outcomes of different models are then aggregated using a LF method (Baraldi et al., 2009; Bonissone et al., 2008). To improve the accuracy of the reconstruction, past signal measurements are used as further input to the models and the reconstruction of the faulty signals is iterated until satisfactory convergence.

The detection and identification of a sensor fault can be achieved by comparing the actual sensor measurements with the signal values estimated by the signal validation and reconstruction model; in this work, the Sequential Probability Ratio Test (SPRT) which considers the statistical properties of the residual, i.e. the difference between the measurements and their estimation, is used.

The proposed approach has been applied to a case study concerning the level and pressure control in the pressurizer of a PWR nuclear power plant. In order to test the effectiveness of the described procedure, faults have been added to sensors whose signals are simulated using a Matlab SIMULINK model of the pressurizer. Upon fault detection, the reconstructed value of the faulty signal is used by the controller to decide the control action. A comparison is made of the control performance obtained when feeding the controller with the measurements coming from the faulty sensor or the reconstructed values.

The remaining parts of the paper are organized as follows. Section 2 states the problems of fault detection, identification and signal reconstruction in the frame of process control; Section 3 describes the signal validation module, recalling briefly the RFSE and LF techniques; Section 4 describes the SPRT technique implemented in the fault detection module; Section 5 presents the results from a set of experiments concerning the control of a simulated PWR pressurizer in presence of faults of the sensors; finally, Section 6 presents the conclusions and describes potential future directions of research.

\section{Sensor fault detection, identification and signal reconstruction for process control}

The objective of a controller is to correct the mismatch between the true values $f_{i_{C}}^{T}$ of $n_{C}$ plant variables and their reference values (setpoints) $f_{i_{C}}^{R e f}$ by establishing and actuating corrective actions. In order to decide the opportune control actions, the controller considers the available information describing the state of the process to be controlled. Let us assume that this information is characterized by the measured values $f_{i_{I}}$ of 
$n_{I}$ plant signals, hereafter called controller input signals. For efficient control of the process, the controller should be fed with the best available estimate of the true values $f_{i_{I}}^{T}, i=1, \ldots, n_{I}$ of each controller input signal. Since a sensor measurement $f_{i_{I}}$ provides an estimate of the true value $f_{i_{I}}^{T}$ of the physical quantity used by the controller, ideally it should be $f_{i_{I}}=f_{i_{I}}^{T}$, but in practice the measurement $f_{i_{I}}$ is affected by random noise and sometimes it also deviates from the true value $f_{i_{I}}^{T}$ because of a sensor fault.

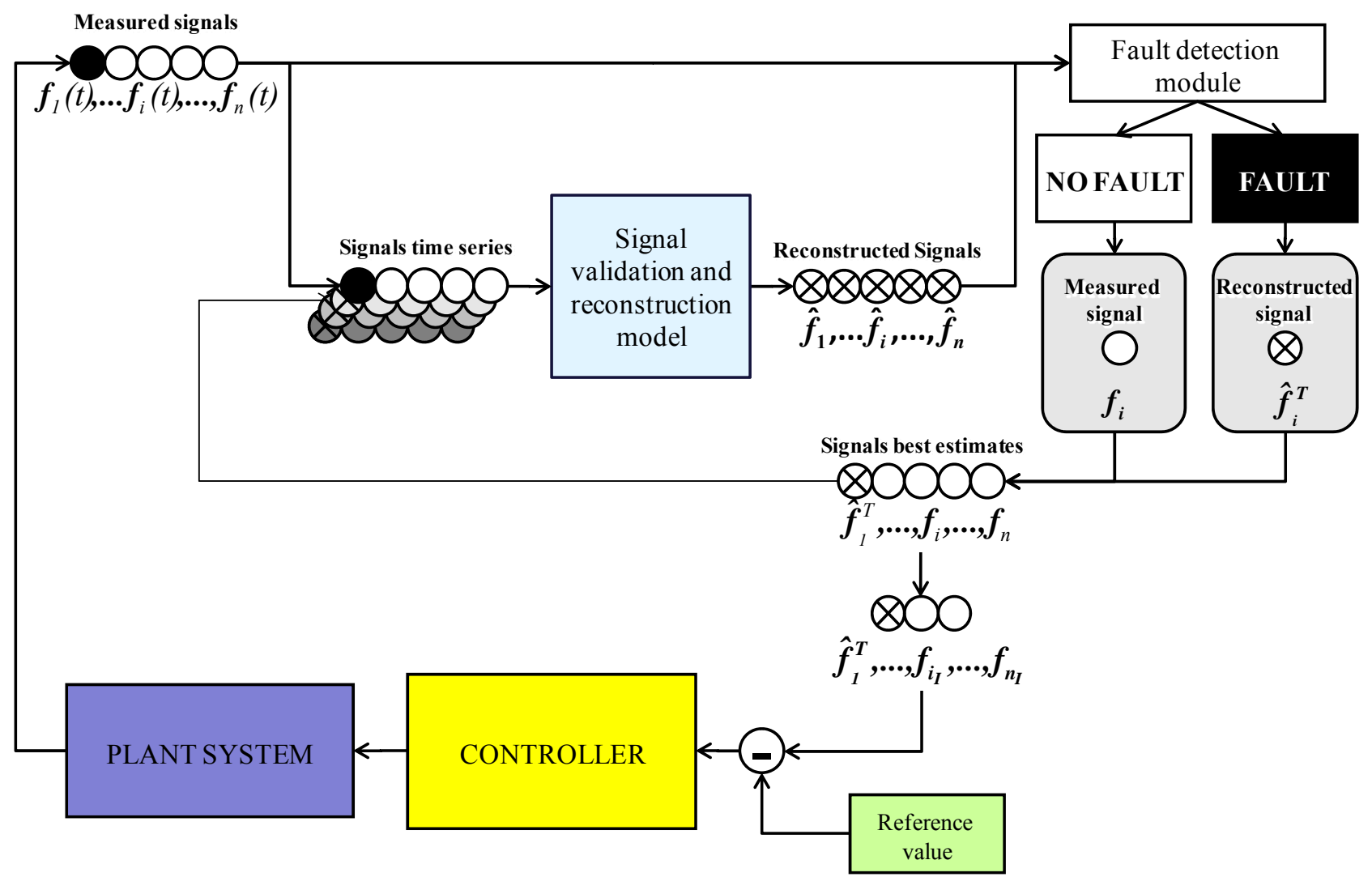

Fig. 1: fault detection, identification and signal reconstruction strategy in case of sensor faults; the black bullet indicates a fault.

Fig. 1 shows the general framework adopted to address the problem of detecting and identifying faults in the sensors measuring the controller input signals $f_{i_{I}}$, and eventually reconstructing their correct values $f_{i_{I}}^{T}$.

Let us assume that $n$ signals of the process under analysis are measured and let $f_{i}$ be the measurement of the generic $i$-th signal, $i=1, \ldots, n$; notice that the $n_{I}$ controller input signals are contained in this set of $n$ signals. The $n$ measured signals $f_{i}$ are processed by a signal validation and reconstruction model which provides a first estimate $\hat{f}_{i}^{l}$ of the true signal value $f_{i}^{T}$ based on the correlation existing between the $n$ measured signals. These correlations are "learned" by the model from a "training" data set $\mathbf{X}_{T R N}$ containing faults-free measurements of the $n$ signals recorded during normal operation of the system. 
In order to catch the dynamic evolution of the system and increase the robustness of the method, the set of measurements $f_{i}$ which are given in input to the signal validation and reconstruction model is constituted not only by the current values $f_{i}(t)$ of the $n$ measured signals, but also by the $n \cdot T$ values $f_{i}(t-1), \ldots, f_{i}(t-T)$ measured in a sliding window of $T$ previous time instants (Fig. 2).

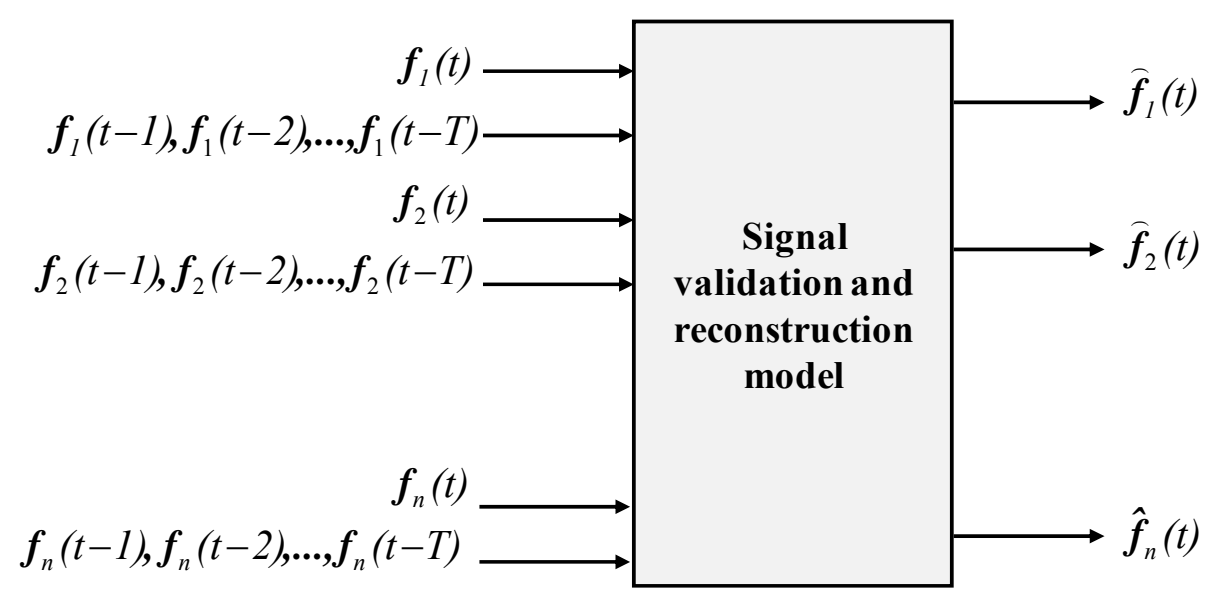

Fig. 2: inputs and outputs of the signal validation and reconstruction model.

The $n$ estimates $\hat{f}_{i}^{l}(t)$ generated by the signal validation and reconstruction model are compared with the measured values $f_{i}(t)$ of the current signals values $f_{i}^{T}(t)$ and the residuals $e_{i}(t)=f_{i}(t)-\hat{f}_{i}^{l}(t)$ are used as input to the fault detection module. By monitoring the evolution of the statistical properties of the residuals $e_{i}$, the conditions of the sensors are verified and an indication about their state of health is obtained. When one or more sensors are diagnosed as faulty, their measurements are not reliable and should be substituted by the estimates produced by the validation and reconstruction model. However, since the estimates $\hat{f}_{i}^{l}$ produced by the signal validation and reconstruction model can be themselves affected by the incorrect values in input, the reconstruction $\hat{f}_{i}^{T}$ of a faulty measurement $f_{i}$ is obtained by iterating the signal reconstruction a number $N^{\text {iter }}$ of times (Chevalier et al., 2009): at each iteration $j$, the $i$-th input to the signal validation and reconstruction model is the reconstructed value $\hat{f}_{i}^{j-1}$ of the faulty signal $f_{i}$ obtained at the previous iteration $j-1$. After the iterations are completed, the reconstructed value $\hat{f}_{i}^{T}(t)$ is used as corrected input to the signal validation and reconstruction model, and also to the controller if $f_{i}$ is one of its inputs, in substitution of the erroneous measurement until maintenance of the faulty sensor is performed.

\section{Signal validation and reconstruction model}

\subsection{The Random Feature Selection Ensemble approach}


Fig. 3 reports a sketch of the flow of modelling for signal reconstruction. A set of $n_{T}=n \cdot(T+1)$ signals $f_{i}(t), f_{i}(t-1), \ldots, f_{i}(t-T), i=1, \ldots, n$ is available for building the signal validation and reconstruction model. Given the typically large value of $n_{T}$, a single model cannot perform the reconstruction task with the desired accuracy and reliability; then, the signal set must be partitioned into subsets, for each of which a reconstruction model is built. Actually, in the ensemble approach here proposed the subsets of signals are overlapping (i.e., two subsets may contain signals which are the same), a PCA model is built for each subset and the reconstruction of a signal contained in different models is obtained by aggregating their outcomes, within an ensemble approach.

In this work, signal grouping is performed by the RFSE technique which consists in randomly sampling from the $n_{T}$ available signals, with replacement, $H$ subsets $\mathbf{F}_{h}, h=1, \ldots, H$, each constituted by $m$ signals (Bryll et al., 2003). This guarantees high signal diversity in the overlapping groups upon which the PCA models are built and allows for rapid construction of the signal groups. Furthermore, randomly selecting the signals in the groups with a reasonable choice of the group size parameters $m$ and $H$, can basically guarantee coverage of all the signals in the ensemble with adequate redundancy (Aly and Atiya, 2006; Bryll et al., 2003).

As mentioned above, the $H$ diverse signal groups generated are used as bases for developing a corresponding number of PCA validation and reconstruction models. To do this, the data set $\mathbf{X}$ of $N$ signal patterns available is partitioned into a training set $\mathbf{X}_{T R N}$ (made of $N_{T R N}$ patterns) and a test set $\mathbf{X}_{T S T}$ (made of $N_{T S T}$ patterns); the former is used to train the individual models, whereas the latter is used to verify the ensemble performance in the signal reconstruction task in order to determine the optimal values of parameters $m$ and $H$ (Baraldi et al., 2009a). The models thus obtained are then used online for the signal validation and reconstruction process.

Each signal $f_{i}$ is present in a number $H_{i}$ of groups and thus a corresponding number of individual PCA models provide its reconstruction. Different methods can be used to aggregate the outcomes of these individual models in the ensemble, in order to get the final reconstructed value. In this work a local fusion strategy has been adopted.

After the ensemble of PCA models has been trained, it can be used to validate and reconstruct the actual sensors measurements: the entire set of $n_{T}$ signals is fed into the ensemble of models which returns their reconstructed values. In particular, each model $h$ receives in input and reconstructs only the signals belonging to the corresponding subset $\mathbf{F}_{h}$. Although the PCA auto-associative models of the ensemble reconstruct in output both current and past values of the measured signals in input, only the reconstructions $\hat{f}_{i}(t)$ of the current measurements are used in the subsequent steps of the control procedure; thus, the aggregation of only the reconstructions $\hat{f}_{i}^{h}(t)$ of the current signals values needs to be performed, with significant savings of computational time. 


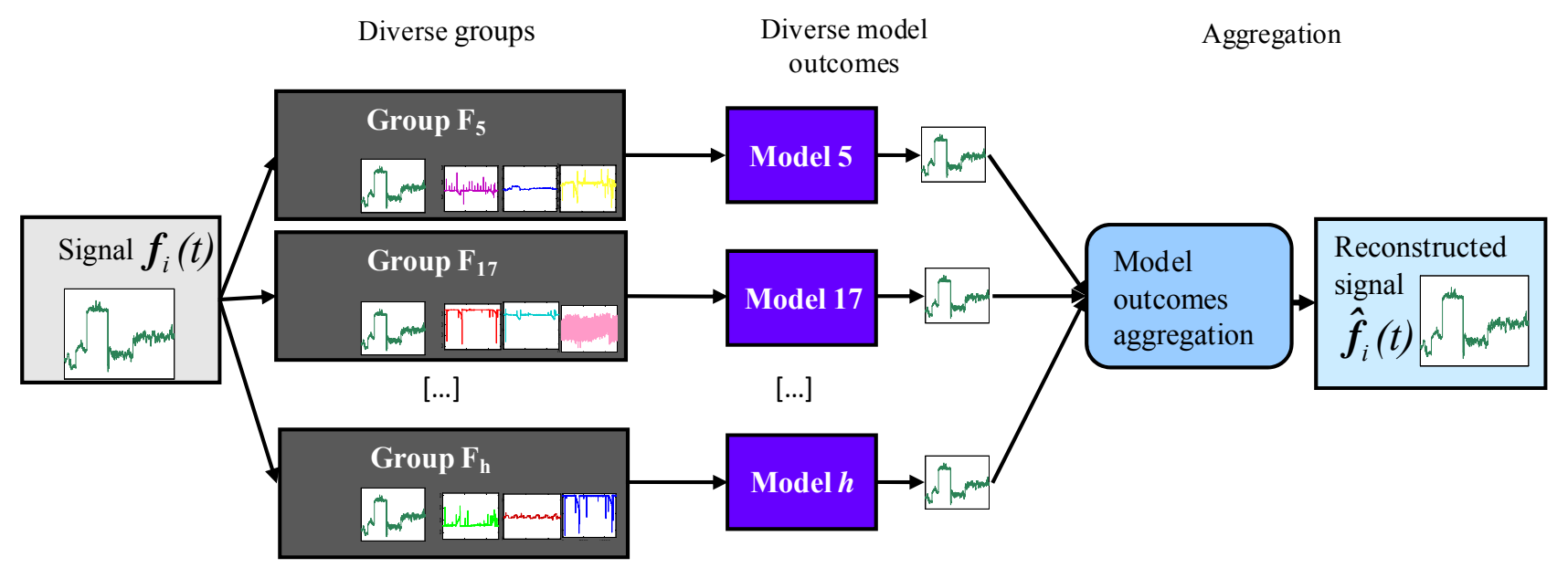

Fig. 3: approach to the reconstruction of signal $f_{i}$ contained in groups $\mathbf{F}_{5}, \mathbf{F}_{17}, \ldots, \mathbf{F}_{h}$.

\subsection{Local fusion strategies for models outcome aggregation}

Different techniques have been proposed for the aggregation of the outcomes of multiple models (Baraldi et al., 2009b; Bonissone et al., 2008). In general, the aggregation of the models outcomes requires to associate a weight $w_{i}^{h}$ and a bias correction $b_{i}^{h}$ to the reconstruction $\hat{f}_{i}^{h}$ of each model $h$. The idea is to correct the values of $\hat{f}_{i}^{h}$ by subtracting the estimated bias $b_{i}^{h}$ and to combine $\hat{f}_{i}^{h}$ with the other models estimates by means of a weighted average:

$$
\hat{f}_{i}=\frac{\sum_{h \mid f_{i} \in \mathbf{F}_{h}} w_{i}^{h} \cdot\left(\hat{f}_{i}^{h}-b_{i}^{h}\right)}{\sum_{h \mid f_{i} \in \mathbf{F}_{h}} w_{i}^{h}}
$$

The most common technique is the simple mean which assigns the same value to all weights and set to zero the bias corrections, i.e. $w_{i}^{h}=\bar{w}$ and $b_{i}^{h}=0, \forall i=1, . ., n$ and $h=1, \ldots, H_{i}$. In (Baraldi et al., 2009b) it has been shown that the ensemble performance can be increased if both the bias corrections $b_{i}^{h}$ and the weights $w_{i}^{h}$ are computed locally, i.e., they vary as a function of the position of the pattern under reconstruction. In this way the two parameters $b_{i}^{h}$ and $w_{i}^{h}$ can account for the variation of models performances in the different regions of the signals space. Fig. 4 shows the overall framework for the reconstruction of the generic signal $f_{i}$ by local fusion of the models outcomes. 


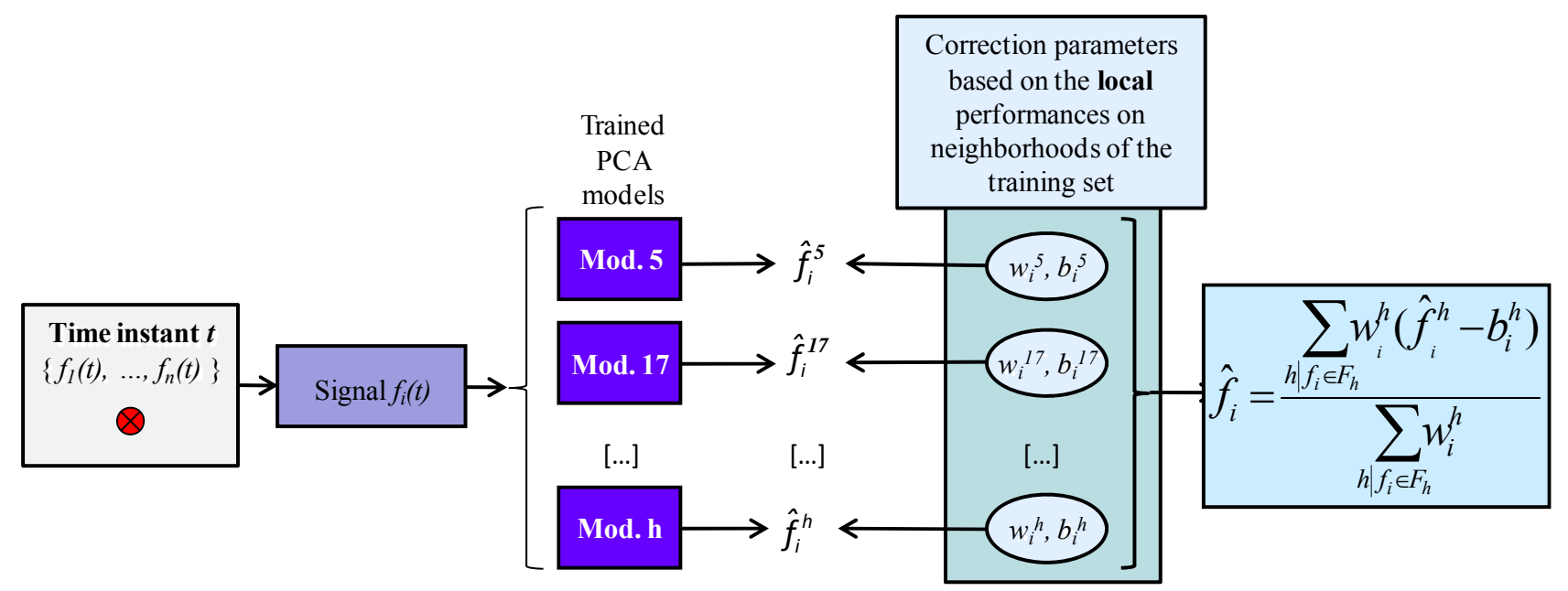

Fig. 4: framework of the locally weighted fusion of model outcomes.

The main steps of the local fusion process are:

1) Retrieve a set $\mathbf{Q}_{T R N}$ of neighbours of the pattern under reconstruction from the set of training patterns. According to the $k$-nn-based neighbourhood approach here adopted (Bonissone et al., 2008), $\mathbf{Q}_{\text {TRN }}$ is formed by the $k$ training patterns nearest to the test pattern (Fig. 5). The optimal number $k$ of nearest neighbours to be considered is obtained verifying the LF performance on the test set $\mathbf{X}_{T S T}$.

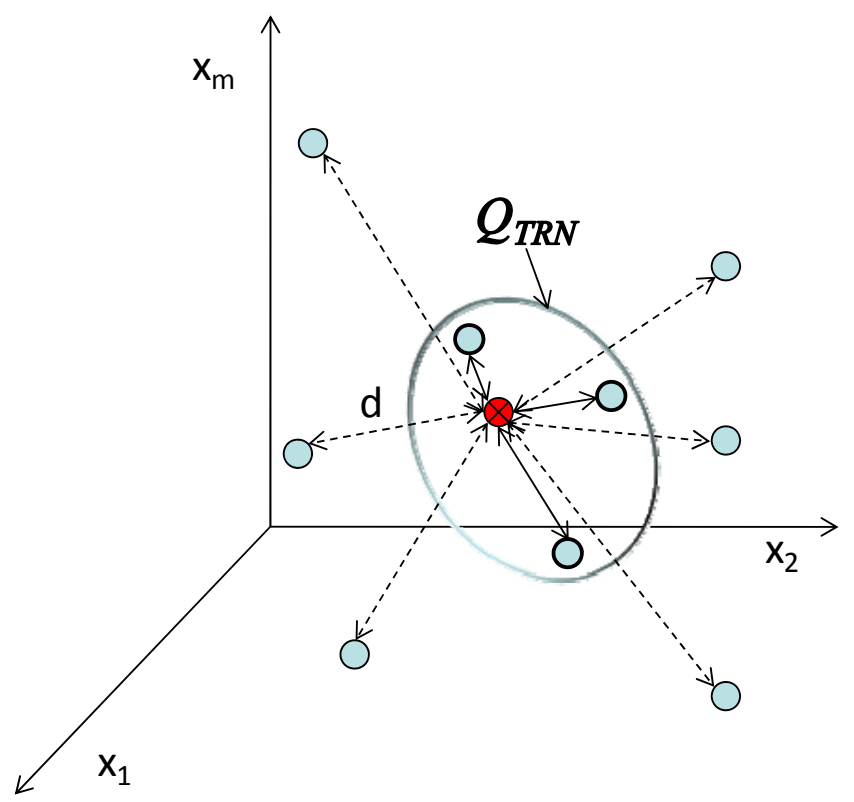

Fig. 5: $k$-nn-based neighborhood. The black circle represents the test pattern; the other circles are training patterns.

2) Associate a weight and a bias to each individual model of the ensemble depending on its reconstruction accuracy on the retrieved neighbours. According to (Baraldi et al., 2009b), the local bias correction $b_{i}^{h}$ and the local weight $w_{i}^{h}$ to be assigned to model $h$ in the reconstruction of signal $f_{i}$ are set equal to: 


$$
\begin{aligned}
& b_{i}^{h}=m e_{i, Q_{T R N}}^{h}=\sum_{Q_{T R N}}\left(\hat{f}_{i}^{h}-f_{i}\right) \\
& w_{i}^{h}=\frac{1}{m a e_{i, Q_{T R N}}^{h}}=\frac{1}{\sum_{Q_{T R N}}\left|\hat{f}_{i}^{h}-f_{i}\right|}
\end{aligned}
$$

where $m e_{i, Q_{T R N}}^{h}$ represents the local mean error and $\operatorname{mae}_{i, Q_{T R N}}^{h}$ the local mean absolute error computed on a different dataset than the one used for the training of the models. The new data set is built by adding an artificial noise to the training data before their reconstruction; in this way, the training error used to estimate the weights will also account for the robustness of the model, thus keeping us from assigning high weights to models whose outstanding performance on the training set is not due to their real quality, but rather derives from overfitting.

3) Aggregate the outputs by using eq. (1), which accounts for the models weights and bias.

\section{Statistical decision logic for fault detection}

The detection of a sensor fault is often achieved by comparison between the actual sensor measurement $f_{i}(t)$ and the measurement estimate $\hat{f}_{i}^{l}(t)$ provided by a signal validation and reconstruction model which reproduces the response of the sensor in non-faulty conditions. Many techniques are based on the monitoring of the residual signal $e_{i}(t)=f_{i}(t)-\hat{f}_{i}^{l}(t)$ (Gross and Kumenik, 1991), for detecting a fault when the residual exceeds a threshold value previously set. To provide the earliest possible indication of a process anomaly, many techniques adopt the sequential probability ratio test (SPRT) (Wald, 1947) to detect changes over time in the statistical characteristics of the residual signal $e_{i}$ (Gross and Kumenik, 1991).

The fault detection module implemented in this work also adopts the SPRT and not just for signalling the occurrence of a fault when the residual exceeds the threshold value, but also to return in output the condition of the sensors, by performing a statistical hypothesis test on the mean and variance of the residuals: at each time step $t$, the null hypothesis $H p_{i}^{0}$, corresponding to the normal, fault-free condition of the $i$-th sensor, is tested against the alternative hypothesis $H p_{i}^{l}$ corresponding to the faulty sensor.

The residual signal $e_{i}$ has the Gaussian distribution $G\left(m_{i}^{j}, \sigma_{i}^{j}\right)$, where $m_{i}^{j}$ and $\sigma_{i}^{j}, j=0,1$ are respectively its mean and standard deviation under the hypothesis $H p_{i}^{j}$. The decision logic which allows choosing between the two hypotheses $H p_{i}^{0}$ and $H p_{i}^{l}$ is based on the value of the log-likelihood ratio $\lambda_{i}(t)$ in the following eq. (4); this is initialized to the value $\lambda_{i}(1)=0$ at the initial time and is updated as follows, as time proceeds (Wald, 1947; Yang et al., 2009):

$$
\lambda_{i}(t)=\lambda_{i}(t-1)+\log \left(\frac{\sigma_{i}^{0}}{\sigma_{i}^{l}}\right)+\frac{\left(e_{i}(t)-m_{i}^{0}\right)^{2}}{\left(\sqrt{2} \sigma_{i}^{0}\right)^{2}}-\frac{\left(e_{i}(t)-m_{i}^{l}\right)^{2}}{\left(\sqrt{2} \sigma_{i}^{l}\right)^{2}}
$$


If the hypothesis $H p_{i}^{0}$ is correct, the fourth term of eq. (4) dominates over the third one and the value of $\lambda_{i}(t)$ decreases during time; vice versa, when the correct hypothesis is $H p_{i}^{l}$ the value of $\lambda_{i}(t)$ increases due to the effect of the third term. In order to decide whether sensor $i$ is faulty or not, a value $\alpha$ is fixed for the probability of false alarm (sensor $i$ is considered faulty although it is in normal condition), and a value $\beta$ is fixed for the probability of missed alarm (sensor $i$ is considered in normal condition although it is faulty). An upper $B_{U}$ and a lower $B_{L}$ thresholds for $\lambda_{i}(t)$ are obtained from $\alpha$ and $\beta$ through eqs. (5) and (6):

$$
\begin{aligned}
& B_{U}=\log [(1-\beta) / \alpha] \\
& B_{U}=\log [\beta /(1-\alpha)]
\end{aligned}
$$

When $\lambda_{i}(t)$ exceeds the value $B_{U}$ a fault to sensor $i$ is detected, whereas the normal condition is diagnosed when the value $B_{L}$ is reached. After one of the two boundaries is reached, the value of the log-likelihood ratio $\lambda_{i}(t)$ is kept constant until its trend is inverted.

Constant bias on the sensor measurement $f_{i}$ can be diagnosed by setting $\sigma_{i}^{l}=\sigma_{i}^{0}$ and $m_{i}^{0}=0$, whereas to detect an increased noise on the sensor measurement one should set $m_{i}^{l}=m_{i}^{0}=0$. The value of $m_{i}^{l}$ in the first case and of $\sigma_{i}^{l}$ in the second depend on the magnitude of the fault one wishes to detect. Too small values of $m_{i}^{l}$ and $\sigma_{i}^{l}$ can lead to false alarms due to the presence of measurement and reconstruction noises also in case of normal sensors conditions, whereas too high values lead to the detection of sensor faults only when they have high magnitudes. The calibration of the SPRT parameters $\alpha, \beta, m_{i}^{l}$ and $\sigma_{i}^{l}$ is performed by a trial and error procedure looking for a trade off between a low rate of false alarms and a low rate of undetected faults.

The fault detection and identification module here implemented is based on the sequential application of the SPRT to all sensors and all types of considered faults (in general bias or noise faults). Although in this work the case of multiple sensor failures is not considered, the proposed procedure can be extended to deal also with this important situation. Also, notice that a fault in the measurement $f_{i}$ may cause some other correlated signals to be incorrectly reconstructed and subsequently falsely identified as faulty too; this phenomenon is called fault propagation and can lead to a high rate of false alarms in signal validation. Then, in case of multiple faults detected by the SPRT, one should verify that they are not a consequence of fault propagation. Different techniques have been proposed to tackle the problem of fault propagation. Within an ensemble approach to signal validation, the technique proposed in (Chenggang and Bingjing, 2006) based on a general sub-grouping technique that uses specially designed intersections between sub-groups to eliminate the false alarms caused by fault propagation seems promising.

\section{Case study}


To verify the applicability of the proposed approach of fault detection, identification and signal reconstruction to the measurements used by a controller for deciding which actions to actuate, a simulation case study regarding the pressurizer of a PWR nuclear power plant has been considered.

\subsection{Pressurizer model}

Fig. 6 is a schematic representation of the pressurizer system for which a Matlab SIMULINK model has been developed, based on the application of the mass and energy conservation equations to the two regions of vapour and liquid; exchanges between the two regions, due to evaporation of liquid and condensation of steam, are taken into account (Kuridan and Beynon, 1998; Todreas and Kazimi, 1990). The system of non linear differential equations describing the model is detailed in the Appendix.

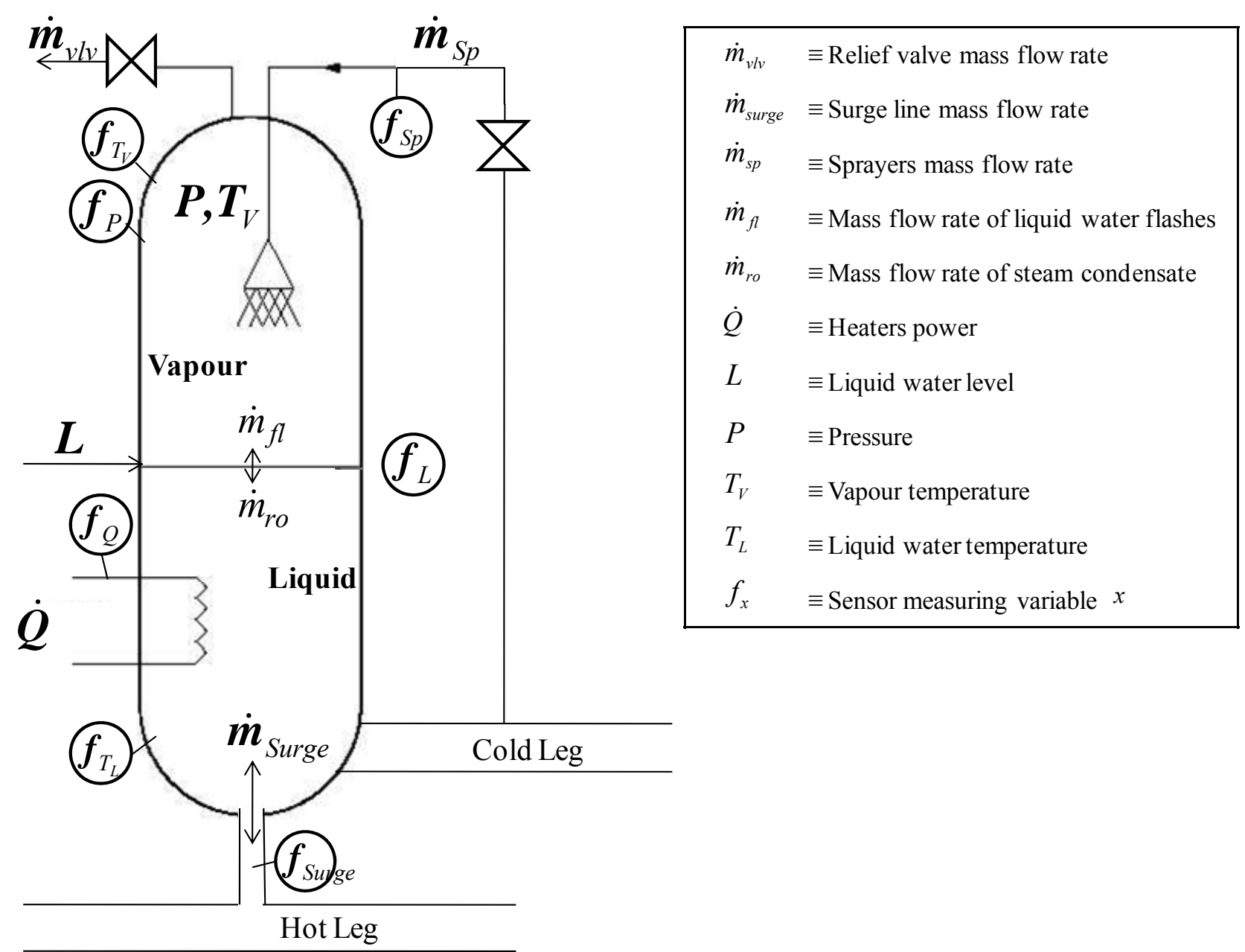

Fig. 6: simplified model of a pressurizer.

The data set $\mathbf{X}$ is built using the signals recorded during different transients, which have been simulated by randomly changing the rate of the surge line mass flow entering the pressurizer. All transients are simulated starting from the initial conditions reported in Table 1. The surge line mass flow rate depends on the reactor 
power; in particular, when the Primary Heat Transport (PHT) system pressure or temperature rise, the higher pressure or the increase of water volume in the PHT circuit push some coolant from this system into the pressurizer (in-surge flow); on the contrary, in case of low PHT system pressure, some liquid moves from the pressurizer into the PHT system circuit (out-surge flow). In this application, the transients have been generated by assuming surge mass flow rates in the range of $[-10 ;+10] \mathrm{kg} / \mathrm{s}$, positive values indicating insurge flow and negative values out-surge flow. In order to represent a realistic situation, the simulations have been carried out based on the operational parameters of a standard PWR pressurizer. Moreover the total mass of water entering or exiting the pressurizer during a surge line mass flow transient has been related to the temperature variations of the coolant in the PHT system.

Table 1

Initial conditions of the pressurizer.

\begin{tabular}{lc}
\multicolumn{2}{c}{ Initial conditions of the pressurizer. } \\
\hline \hline Level & $7.221 \mathrm{~m}$ \\
Liquid Temperature & $342.1^{\circ} \mathrm{C}$ \\
Vapour Temperature & $342.3^{\circ} \mathrm{C}$ \\
Pressure & $150.0 \mathrm{bar}$ \\
\hline
\end{tabular}

During the simulations, the $n=7$ sensors measuring the signals reported in Table 2 have been considered.

Table 2

Measured signals.

\begin{tabular}{lcc}
\multicolumn{3}{c}{ Measured signals. } \\
\hline PHYSICAL QUANTITY & SYMBOL & MEASURED SIGNAL \\
\hline \hline Liquid water level & $L$ & $f_{L}$ \\
Liquid water temperature & $T_{L}$ & $f_{T_{L}}$ \\
Vapour temperature & $T_{V}$ & $f_{T_{V}}$ \\
Pressure & $P$ & $f_{P}$ \\
Spray mass flow rate & $\dot{m}_{s p}$ & $f_{s p}$ \\
Heaters power & $\dot{Q}$ & $f_{\dot{Q}}$ \\
Surge line mass flow rate & $\dot{m}_{\text {surge }}$ & $f_{\text {surge }}$ \\
\hline
\end{tabular}

A block diagram of the model identifying the inputs, state of the system, sensors outputs and controller variables is shown in Fig. 7. 


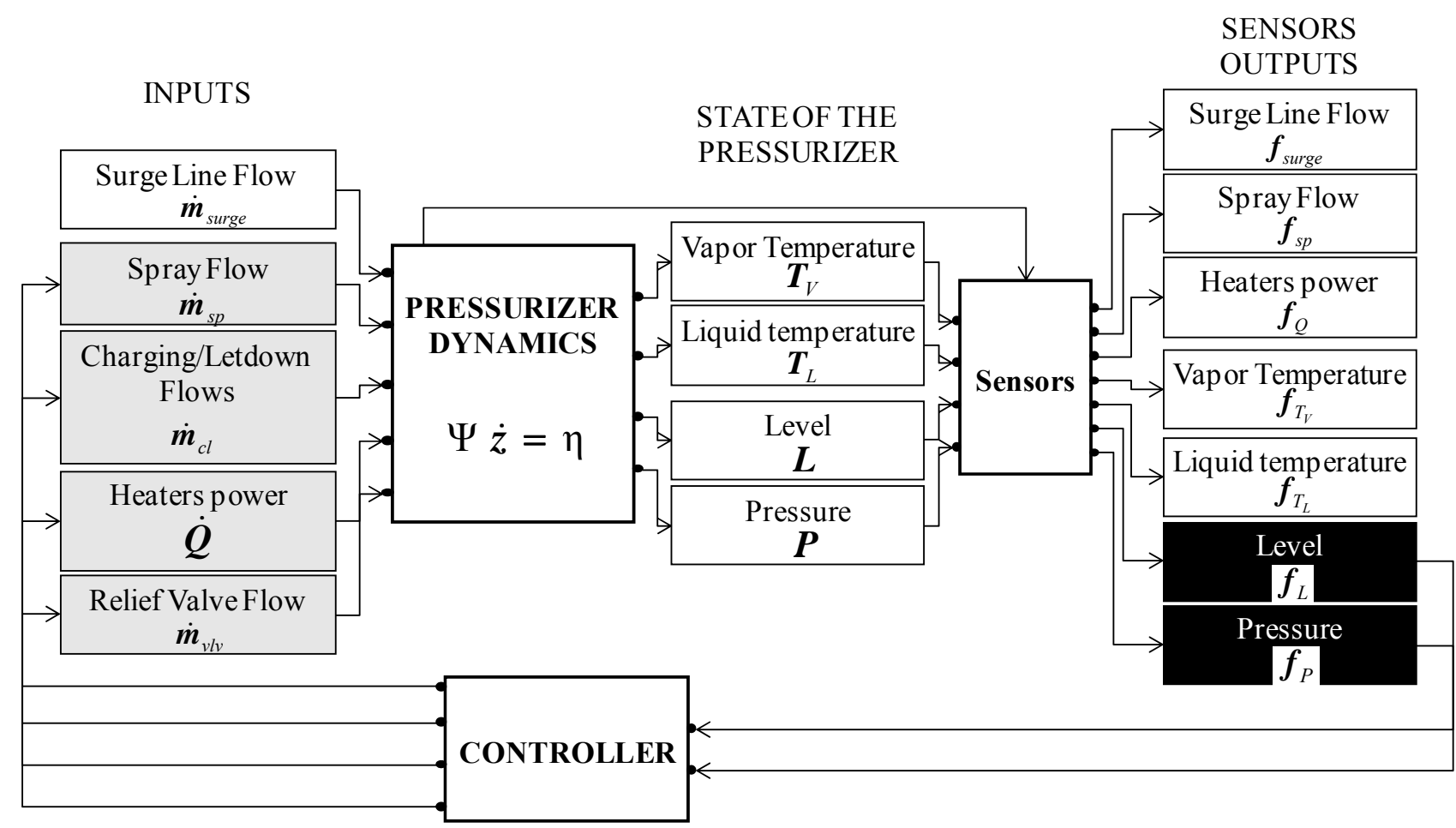

Fig. 7: inputs, state, controller outputs (gray), controller inputs (black) and sensors outputs of the SIMULINK model of the pressurizer.

In order to test the method on pseudo-realistic data, white noise has been added to each signal according to engineering considerations on the sensors accuracy (Hashemian, 2004; Hashemian, 2006; Johnson, 2008). Table 3 reports the standard deviations of the considered noises.

Table 3

Standard deviation of sensors noise.

\begin{tabular}{lc}
\multicolumn{2}{c}{ Standard deviation of sensors noise. } \\
\hline & NOISE STANDARD \\
& DEVIATION \\
\hline \hline Level Sensor & $\pm 0.01 \mathrm{~m}$ \\
Pressure Sensor & $\pm 0.5 \mathrm{bar}$ \\
Flow Sensor & $\pm 0.2 \mathrm{~kg} / \mathrm{s}$ \\
Power Sensor & $\pm 50 \mathrm{~kW}$ \\
\hline
\end{tabular}

\subsection{Controller model}

In this application, the control of the level $L$ and the pressure $P$ in the pressurizer is achieved through the feedback control scheme shown in Fig. 7, which reproduces the scheme used in a standard PWR pressurizer. The pressure $f_{P}$ and level $f_{L}$ are the controlled signals as well as the controller input signals; the sprayers mass flow rate $\dot{m}_{s p}$, the relief valve mass flow rate $\dot{m}_{v / v}$, the heaters power $\dot{Q}$ and the charging/letdown mass flows rates $\dot{m}_{c l}$ are the controller outputs. More details on the characteristics of the controller implemented in this application are described in the Appendix. 
Fig. 8 reports the noise-free evolution of the seven measured variables during one of the simulated transients. The initial state of the system is varied by an out-surge mass flow rate $\dot{m}_{\text {surge }}=-6 \mathrm{~kg} / \mathrm{s}$ lasting for $175 \mathrm{~s}$ and removing from the pressurizer a total mass $M=970 \mathrm{~kg}$ corresponding to a variation of the PHT system temperature of $-2{ }^{\circ} \mathrm{C}$. The out-surge flow produces a decrease in the pressure and consequently the heaters are turned on so that the pressure decrease is slowed down. When the out-surge flow ends, the heaters drive the pressure back to its reference value of $f_{P}^{R e f}$. The loss of liquid in the pressurizer causes the water level to decrease until a stabilized value of $L=6.68 \mathrm{~m}$ is reached. The controller intervenes only when the absolute value of difference $\varepsilon_{L}=f_{L}^{R e f}-f_{L}$ between the level reference value and level signal is greater than a tolerated error band $B_{L}^{\varepsilon}=2 \mathrm{~m}$, which is much larger than the pressure tolerated error band $B_{P}^{\varepsilon}=0.2$ bar. In this case, the error $\varepsilon_{L}$ is equal to $0.06 \mathrm{~m}$, as the reference value for the level is $f_{L}^{R e f}=6.74 \mathrm{~m}$ for a PHT temperature decrease of $-2{ }^{\circ} \mathrm{C}$; since $\left|\varepsilon_{L}\right|<B_{L}^{\varepsilon}$ and the control on the level does not intervene.
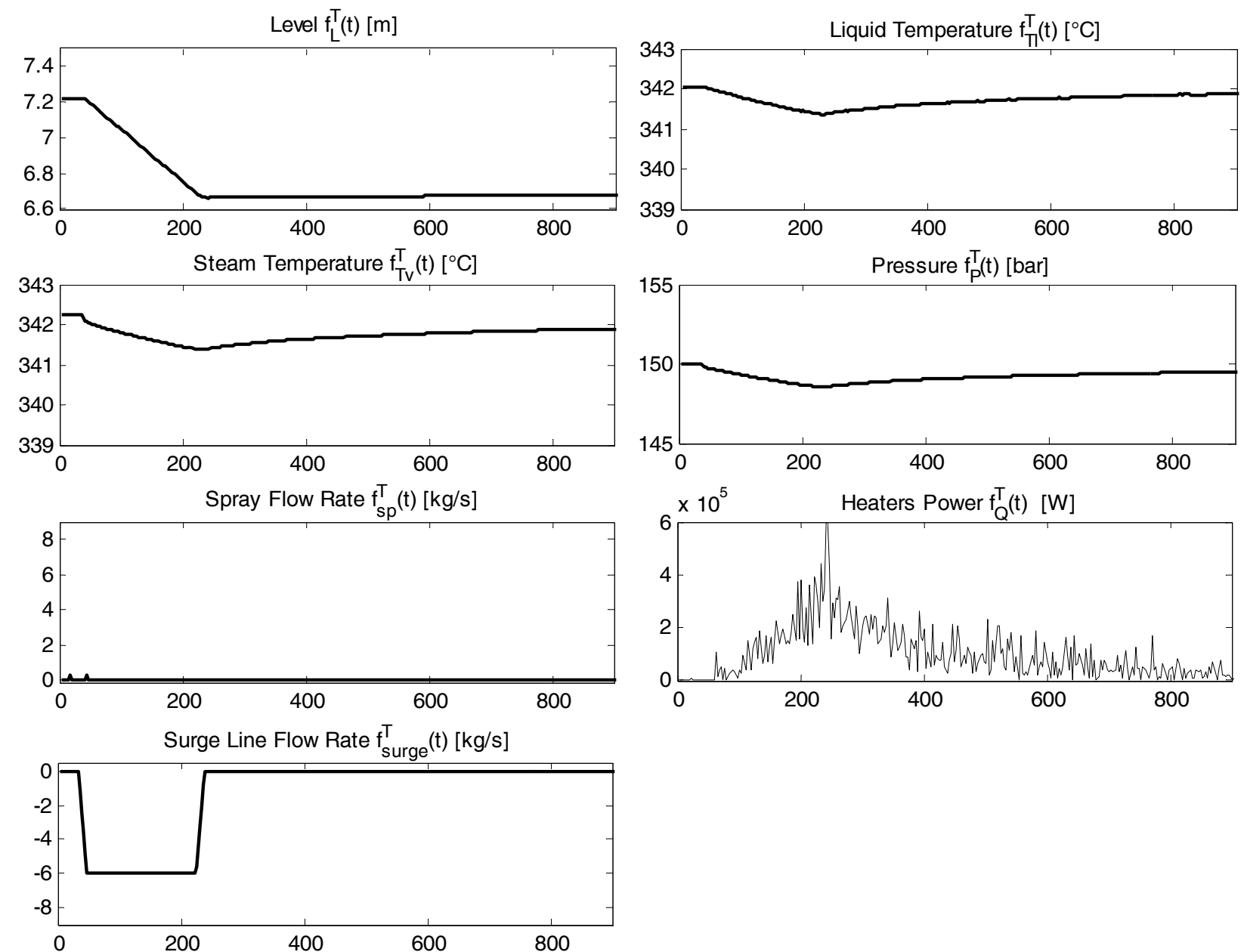

Fig. 8: true noise-free values $f_{i}^{T}$ of the seven measured variables evolving during a plant transient. 
The actual values of the signals $f_{i}$ measured by the seven sensors during this transient are shown in Fig. 9 . In this case the measurement noises received by the controller cause the irregular shape of the heaters power controller output signal. Notice that the controller inputs signals $f_{i_{I}}$ are the pressure and level signals $f_{P}$ and $f_{L}$ of Fig. 9 and not those noise-free of Fig. 8, that represent the true values $f_{P}^{T}$ and $f_{L}^{T}$ of the physical quantities.
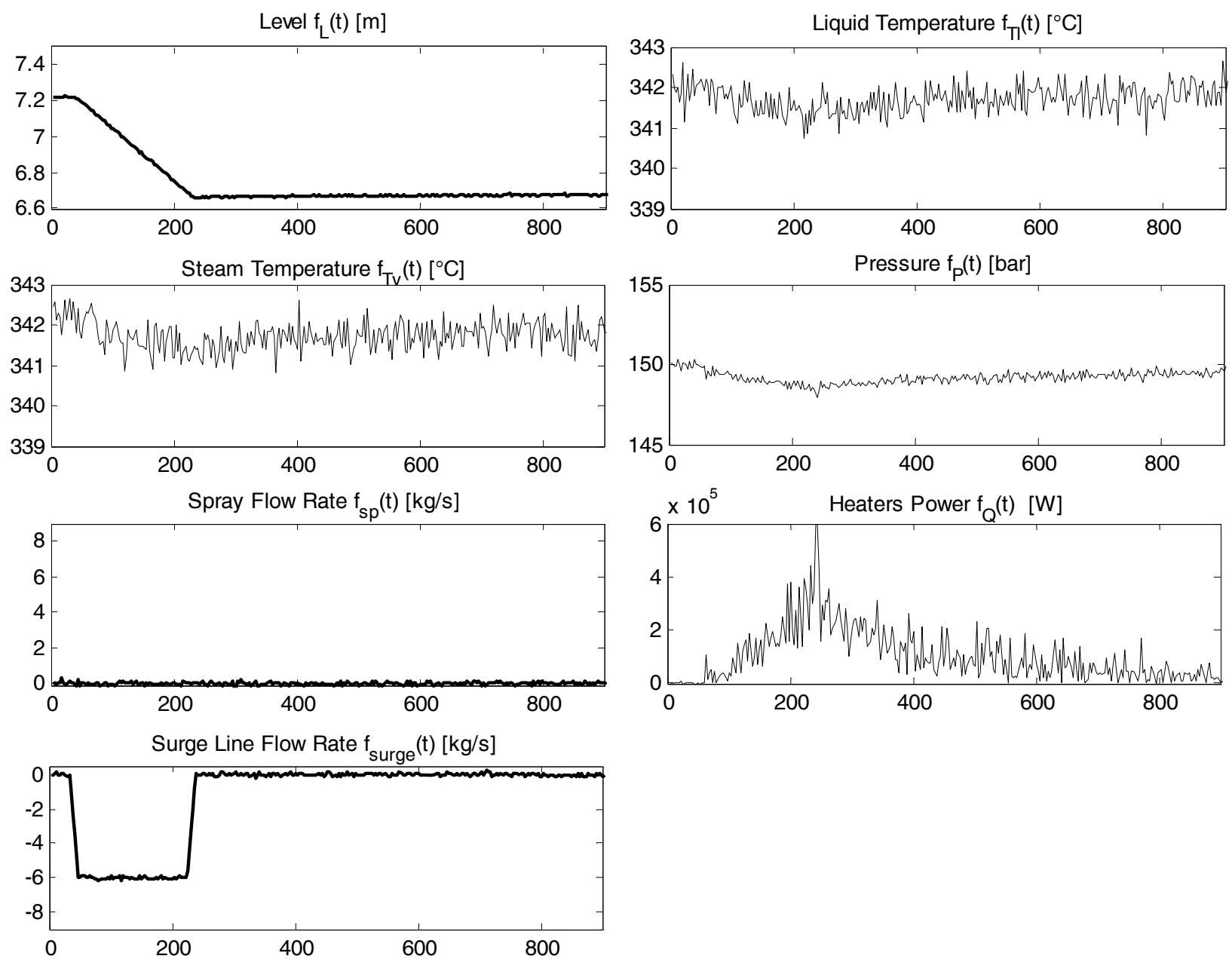

Fig. 9: signal values $f_{i}$ actually measured (with noise) during the transient of Fig. 8.

\subsection{Simulation of sensor faults}

When a fault affects the pressure or the level measurements, the controller receives incorrect input signals which may lead to an actuation decision different from the one it would take in absence of sensor faults. Let $f_{i_{C}}^{T}(t)$ represent the evolution of the $i_{C}$-th controlled signal if all sensors are in normal conditions and $f_{i_{C}, F\left(i_{I}\right)}^{T}(t)$ its evolution if there is a fault of the controller input signal $f_{i_{I}}$; notice that both signals $f_{i_{C}}^{T}(t)$ 
and $f_{i_{C}, F\left(i_{I}\right)}^{T}(t)$ indicate the true value of the $i_{C}$-th controlled signal in a transient but they result from two different controller decision strategies, because based on fault-free and faulty information respectively.

In this case study, pressure and level sensor faults are simulated by adding a constant bias of magnitude $M_{B}$ to the true value $f_{i}^{T}$ of the signal. For the entire duration of the fault, the measurement produced by sensor $i$, which usually is given by $f_{i}=f_{i}^{T}+$ noise, will then be equal to $f_{i}=f_{i}^{T}+M_{B}+$ noise.

To illustrate the consequences of a fault on a sensor measuring a controller input signal, the effects on the system of the fault reported in Table 4 are considered.

Table 4

Pressurizer condition during the faulty transient simulated.

\begin{tabular}{cc}
\hline Pressurizer condition during the faulty transient simulated. \\
TRANSIENT TYPE & Out-surge [Fig. 8, Fig. 9] \\
SURGE LINE MASS FLOW RATE & $\dot{m}_{\text {surge }}=+6 \mathrm{~kg} / \mathrm{s}$ \\
FAULTY SENSOR & Pressure sensor \\
TYPE OF FAULT & Constant bias \\
FAULT MAGNITUDE & $M_{B}=4.5 \mathrm{bar}$ \\
BEGINNING TIME & $t=50 \mathrm{~s}$ \\
\hline
\end{tabular}

Fig. 10 compares the evolution of the pressure $f_{P}^{T}(t)$ (upper) and the level $f_{L}^{T}(t)$ (bottom) signals in normal sensor conditions (left) to the evolution of the same two signals $f_{P, F(P)}^{T}(t)$ and $f_{L, F(P)}^{T}(t)$ in the case of the pressure sensor fault of Table 4 (right). Fig. 11 compares, in the same case, the behaviours of the two controller output variables used to control the process, i.e. the sprayers mass flow rate (upper) and the heaters power (bottom). 

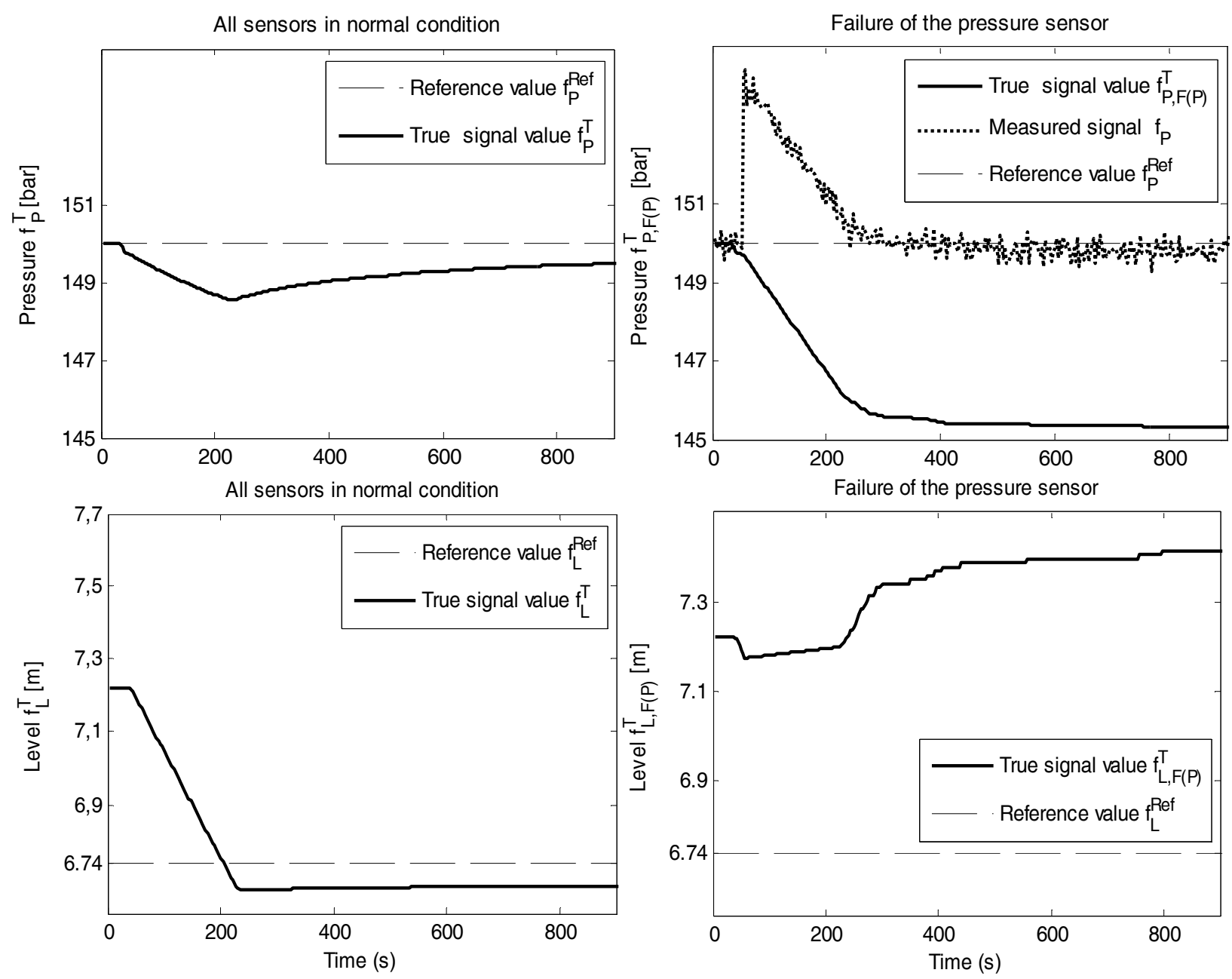

Fig. 10: evolution of the pressure (upper) and of the level (bottom) in the pressurizer when all the sensors are in normal conditions (left), and when a constant bias fault of +4.5 bar on the pressure measurement is simulated and the measured signal $f_{i_{I}}$ is used as input to the controller (right). 

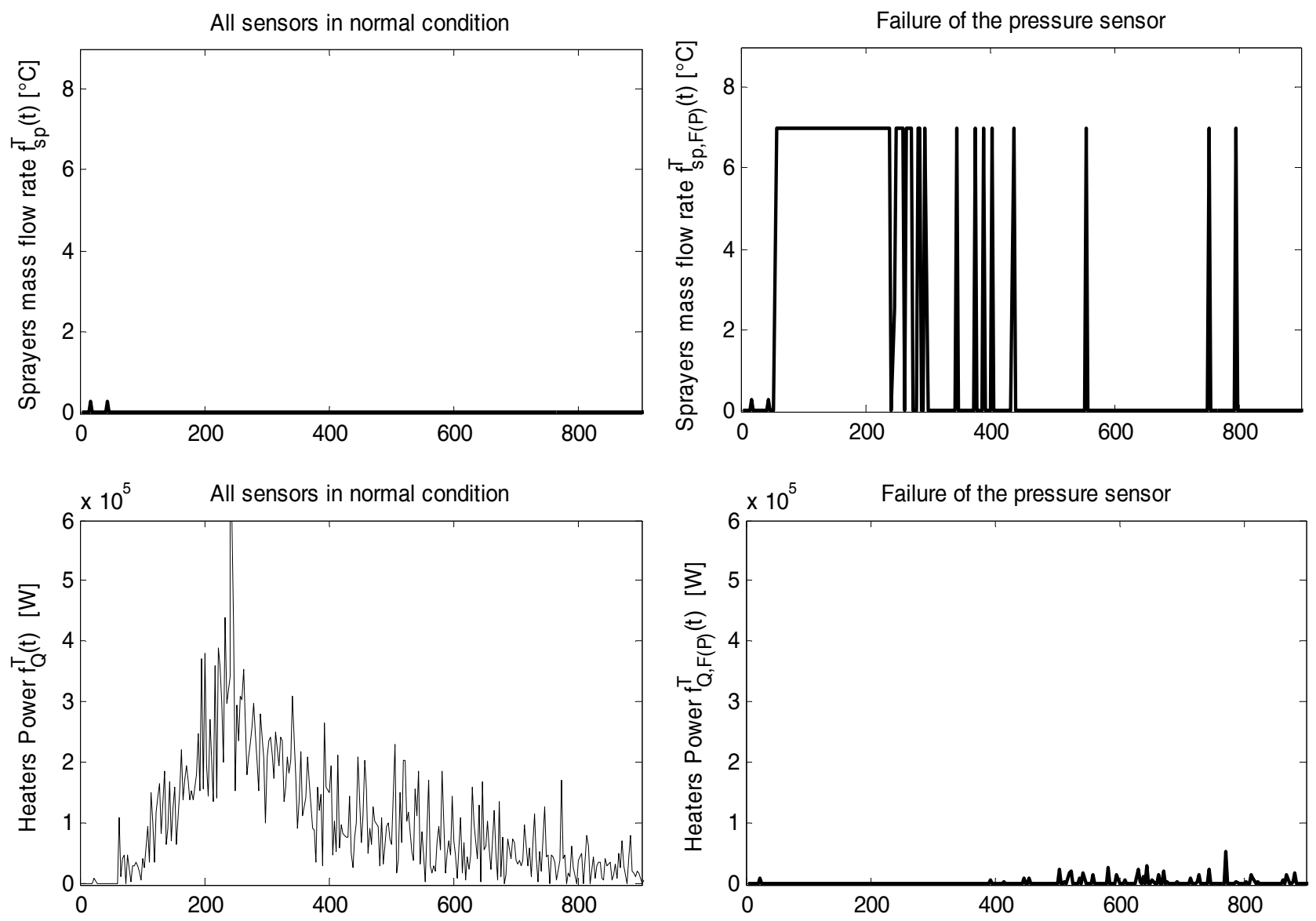

Fig. 11: evolution of the sprayers mass flow rate (upper) and of the heaters (bottom) in the pressurizer when all the sensors are in normal conditions (left), and when a constant bias fault of +4.5 bar on the pressure measurement is simulated and the measured signal $f_{i_{I}}$ is used as input to the controller (right).

Notice that at $t=50 \mathrm{~s}$, when there is the pressure sensor fault, the controller believes (wrongly) that the pressure has exceeded its reference value $f_{P}^{R e f}$; therefore, instead of starting the heaters at $t=60 \mathrm{~s}$ as it would have done in normal sensors conditions (Fig. 11, bottom left), it turns on the sprayers in order to decrease the pressure (Fig. 11, upper right). Consequently, the undesired pressure decrease is accelerated by the wrong control decision. At the end of the plant transient, when the out-surge stops, the pressure stabilizes at a value which is lower than the reference of a quantity comparable with the amplitude of the sensor bias. At the same time, the total mass of liquid introduced in the pressurizer by the sprayers increases the level of the liquid water. At the end of the transient, the absolute value of the difference between the level reference value $f_{L}^{R e f}=6.74 \mathrm{~m}$ and the actual level value $f_{L}^{T}=7.46 \mathrm{~m}$ is $\left|\varepsilon_{L}\right|=0.72 \mathrm{~m}$, which is still lower than the tolerated error band $B_{L}^{\varepsilon}$, and thus no action on the charging/letdown mass flows is started to correct this mismatch. 
This example demonstrates that the controller can actually take wrong decisions when its inputs are altered by a sensor fault.

\subsection{Application of the fault detection and signal reconstruction methodology}

In this Section, the developed model for the fault detection, identification and signal reconstruction is described and applied to the case study under analysis.

\subsubsection{Signal validation}

Since the pressurizer operation strongly depends from the pressure and temperature of the PHT system, in order to build a more accurate signal validation model the information coming from the seven sensors of the pressurizer should be integrated by information related with the PHT system state. In this case study, since only the pressurizer and not the complete reactor has been simulated, the variations occurring outside the pressurizer are taken into account by considering an additional signal numerically computed as $f_{M}(t)=\int_{0}^{t} f_{\text {surge }}(\tau) d \tau$, which represents the transfer of mass between the PHT system and the pressurizer during a transient.

The set of $n_{T}=n(T+1)$ signals $f_{i}(t), f_{i}(t-1), \ldots, f_{i}(t-T), T=9, i=1, \ldots, n, n=8$ which are given as input to the validation model is made of current and past values of the measured signals listed in Section 5.1.1 plus the present and past values of $f_{M}$ (Fig. 12).

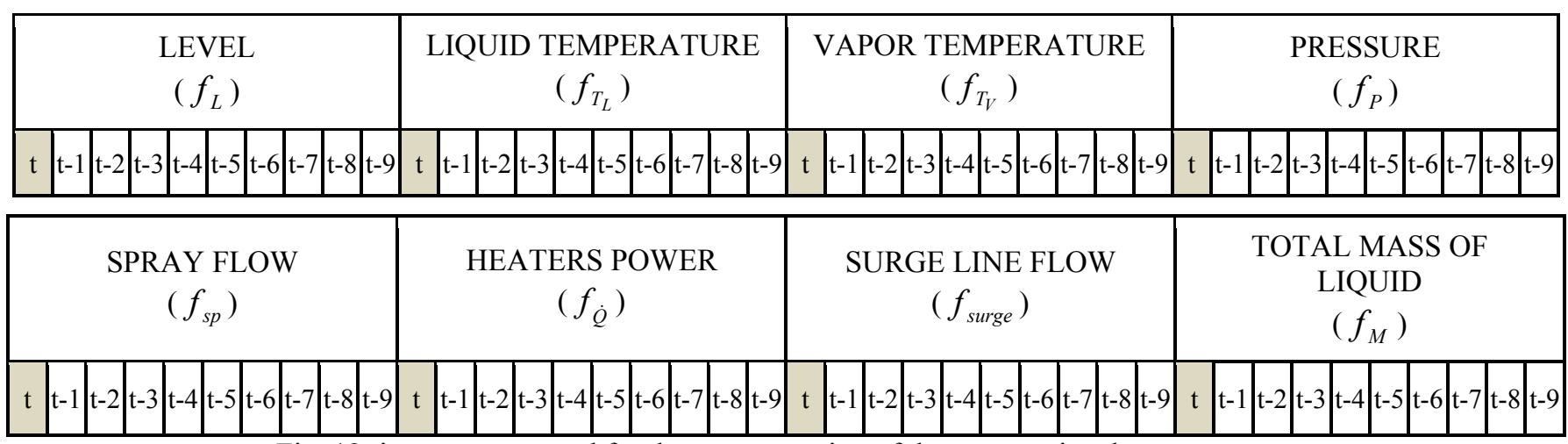

Fig. 12: input pattern used for the reconstruction of the current signal measurement.

A set of 70 faults-free transients have been simulated and used for the training of the PCA models, whereas 10 distinct transients have been used for testing the performance of the proposed approach in order to optimize the parameters $m, H$ and $k$. The final asset consists of $H=32$ subsets $\mathbf{F}_{h}$, each one containing $m=25$ signals; for each signal, 10 different models reconstructions are aggregated by the LF strategy with parameters $w_{i}^{h}$ and $b_{i}^{h}$ computed on the basis of $k=30$ neighbouring training patterns. Notice that in order 
to reduce the computational time, redundancies higher than 10 have not been considered although they may increase the ensemble performance.

Fig. 13 illustrates how the signal validation strategy presented in Section 2 has been applied to the pressurizer case study. The set of seven current measured signals $f_{L}, f_{T_{L}}, f_{T_{V}}, f_{P}, f_{s p}, f_{\dot{Q}}, f_{\text {surge }}$ of Table 2 and the additional artificial signal $f_{M}$ are completed with their past 9 measurements and sent to the signal validation model. For each one of the seven measurements, the reconstruction $\hat{f}_{i}^{1}, i=1, \ldots, 7$ is produced by the ensemble model and compared with the measured value $f_{i}$. The residual $e_{i}$ is sent to the fault detection module where it is processed by means of the SPRT technique. The faults eventually detected are corrected by iterating $N^{\text {iter }}=5$ times the reconstruction of the signal. The final reconstruction $\hat{f}_{i}^{5}$ is the best available estimate $\hat{f}_{i}^{T}$ of the faulty signal true value $f_{i}^{T}$ and it is memorized in place of the measurement $f_{i}$. If the detected fault concerns the pressure or the level signals, the controller receives in input the corresponding reconstructed signal $\hat{f}_{P}^{T}$ or $\hat{f}_{L}^{T}$. In any case, the reconstruction $\hat{f}_{i}^{T}$ of the faulty signal is also included in place of the measured value $f_{i}$ into the patterns sent to the signal validation model during the next $T$ time instants.

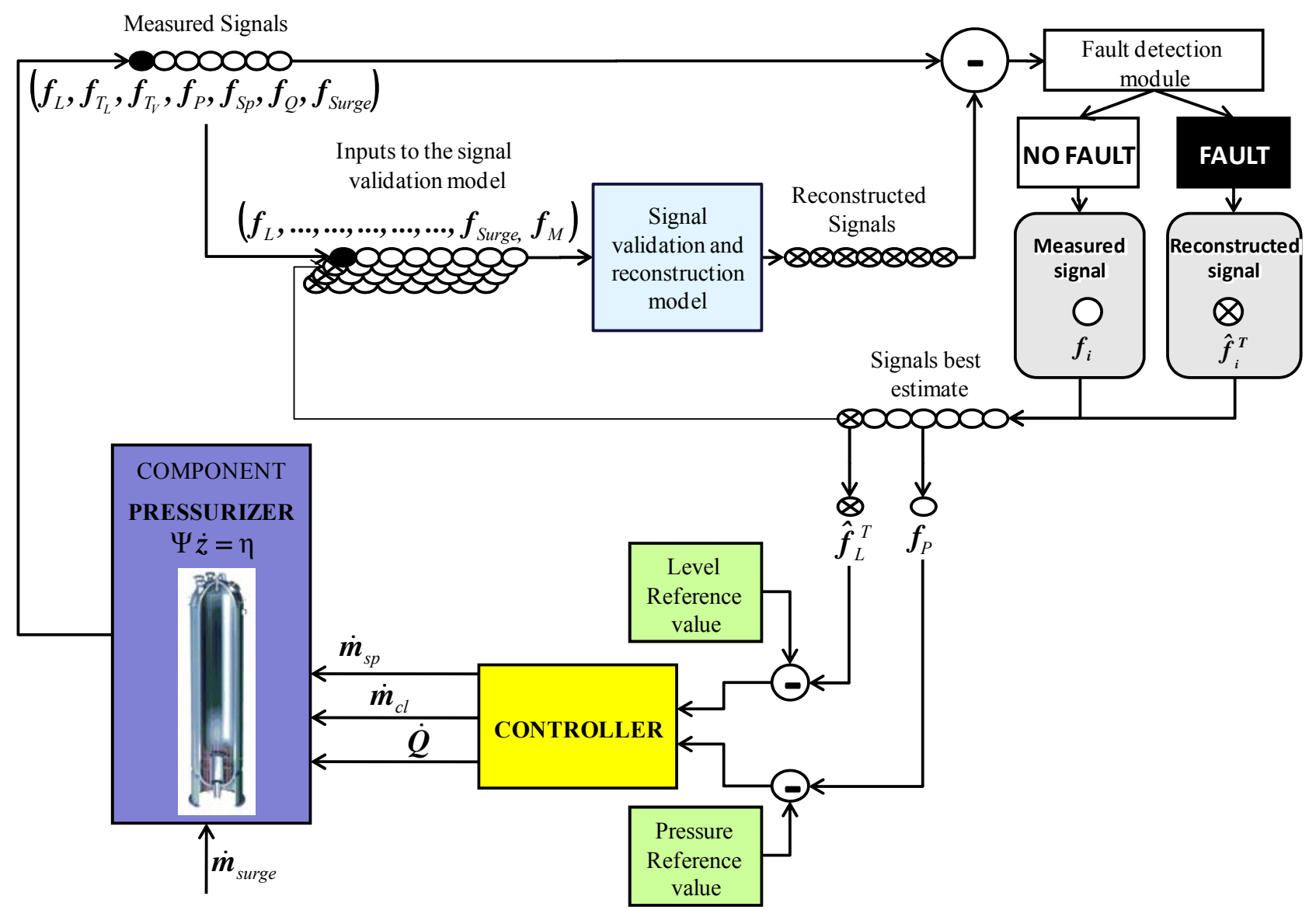

Fig. 13: signal reconstruction strategy for control of the pressurizer of a PWR in the case of a fault of the level sensor (black dot). 


\subsubsection{The fault detection and identification module}

The signal validation model produces a reconstruction $\hat{f}_{i}^{1}, i=1, \ldots, 7$ of each measured signal, which allows the fault detection and identification module to recognize the presence of sensor faults (bias or noise), by applying the SPRT sequentially to each sensor signal.

Since in this work we only consider constant bias faults, the standard deviations of all the residuals $e_{i}$, $i=1, \ldots, n$ are set at the same value $\sigma_{i}^{l}=\sigma_{i}^{0}=1$ whereas their mean values under the hypothesis $H p_{i}^{0}$ are $m_{i}^{0}=0$. The SPRT parameters $\alpha, \beta$ and $m_{i}^{l}$ have been calibrated by a trial and error procedure performed on the test set $\mathbf{X}_{T S T}$ after addition of artificial faults, with the goal of compromising between the objective of detecting faults of an as small as possible magnitude and in a short time after their occurrence, and that of achieving a low rate of false alarms. The values assigned to the mean values of the residuals under hypothesis $H p_{i}^{l}$ are $m_{P}^{l}=1$ for a pressure sensor fault, $m_{L}^{l}=0.7$ for a level sensor fault and $m_{i}^{l}=4$ for all other signals faults. This choice implies a small sensibility of the fault detection module in case of faults affecting signals other than the pressure or the level, but it reduces the risk of false alarms due to fault propagation. Finally, the false and missed alarm probabilities $\alpha$ and $\beta$ are set to the values $\alpha, \beta=0.005$, for all sensors.

Fig. 14 shows the evolution of $\lambda_{P}(t)$ in the case of the pressure sensor fault of Table 4 , when the signal validation strategy is applied; one can see that during the first time instants, the value of $\lambda_{P}$ resulting from the SPRT applied to the pressure sensor decreases until it reaches at $t=19 \mathrm{~s}$ the lower bound $B_{L}=-5.29$ and thus one can say that the pressure sensor is in normal condition with a degree of confidence $(1-\beta)=99.5 \%$; at time $t=50 \mathrm{~s}$ the value of $\lambda_{P}$ quickly increases up to the upper bound $B_{U}=5.29$ and the fault is detected with a probability of $\alpha=0.5 \%$ of being a false alarm; from the detection time on, the controller receives in input the reconstructed value $\hat{f}_{P}^{T}$ of the pressure signal. 


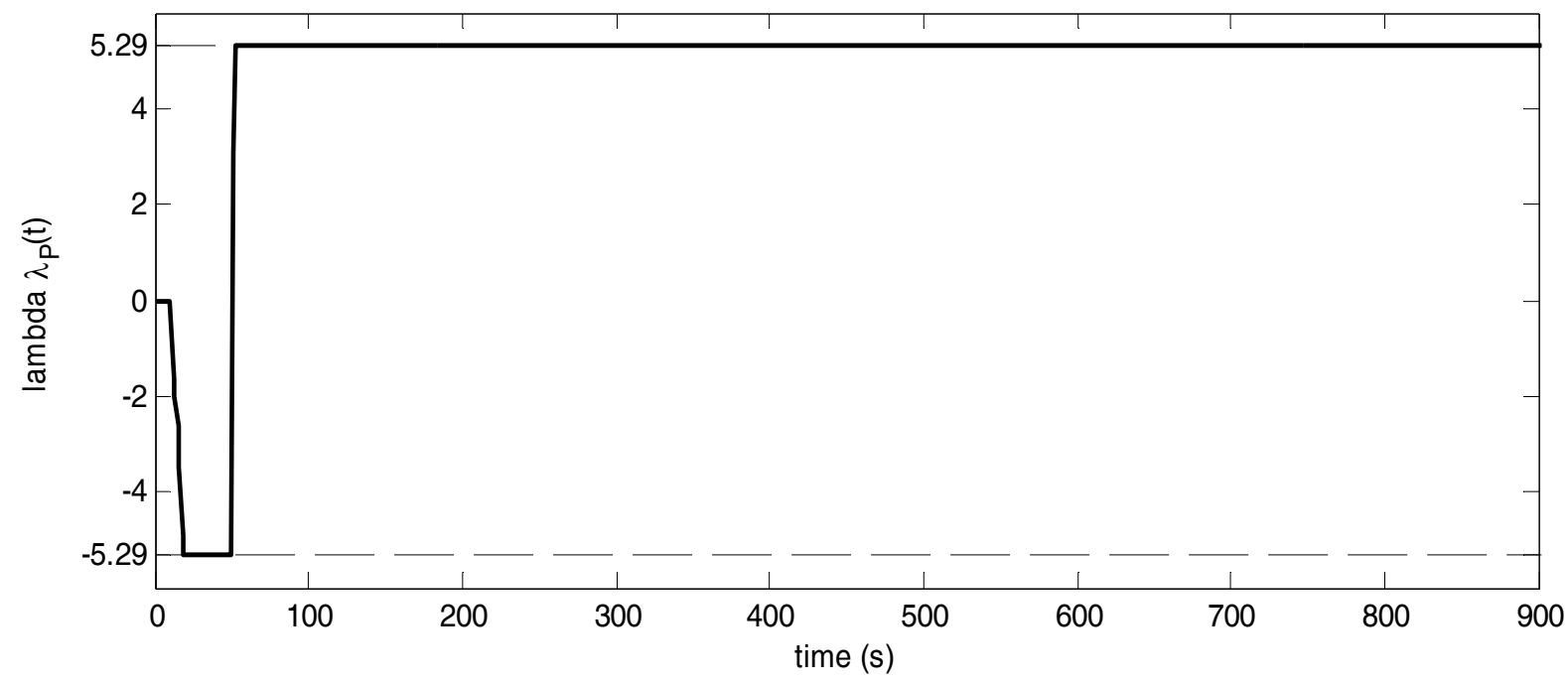

Fig. 14: evolution of $\lambda_{P}(t)$ during the transient of Table 4 where a fault is added to the pressure sensor measurement.

\subsubsection{The signal reconstruction module}

Once again the pressure sensor fault of Table 4 is used to illustrate the improvements which can be obtained by reconstructing the faulty signals after the fault has been detected by the SPRT. Fig. 15 compares the evolution of the true pressure and level signals values, $f_{P}^{T}(t)$ and $f_{L}^{T}(t)$ respectively, in the case of normal sensors conditions (left) and in the case of the fault affecting the pressure measurement, when applying the fault detection, identification and signal reconstruction modules (right). Fig. 16 compares, in the same case, the behaviours of the heaters; the sprayers mass flow rate is in both cases almost always zero, and thus it is not shown here. Notice that, even if a sensor fault has occurred, the evolutions of all signals and in particular of the true pressure and level signals, $f_{P, F(P)}^{T}(t)$ and $f_{L, F(P)}^{T}(t)$, are very similar to those obtained in case of normal sensors conditions. 

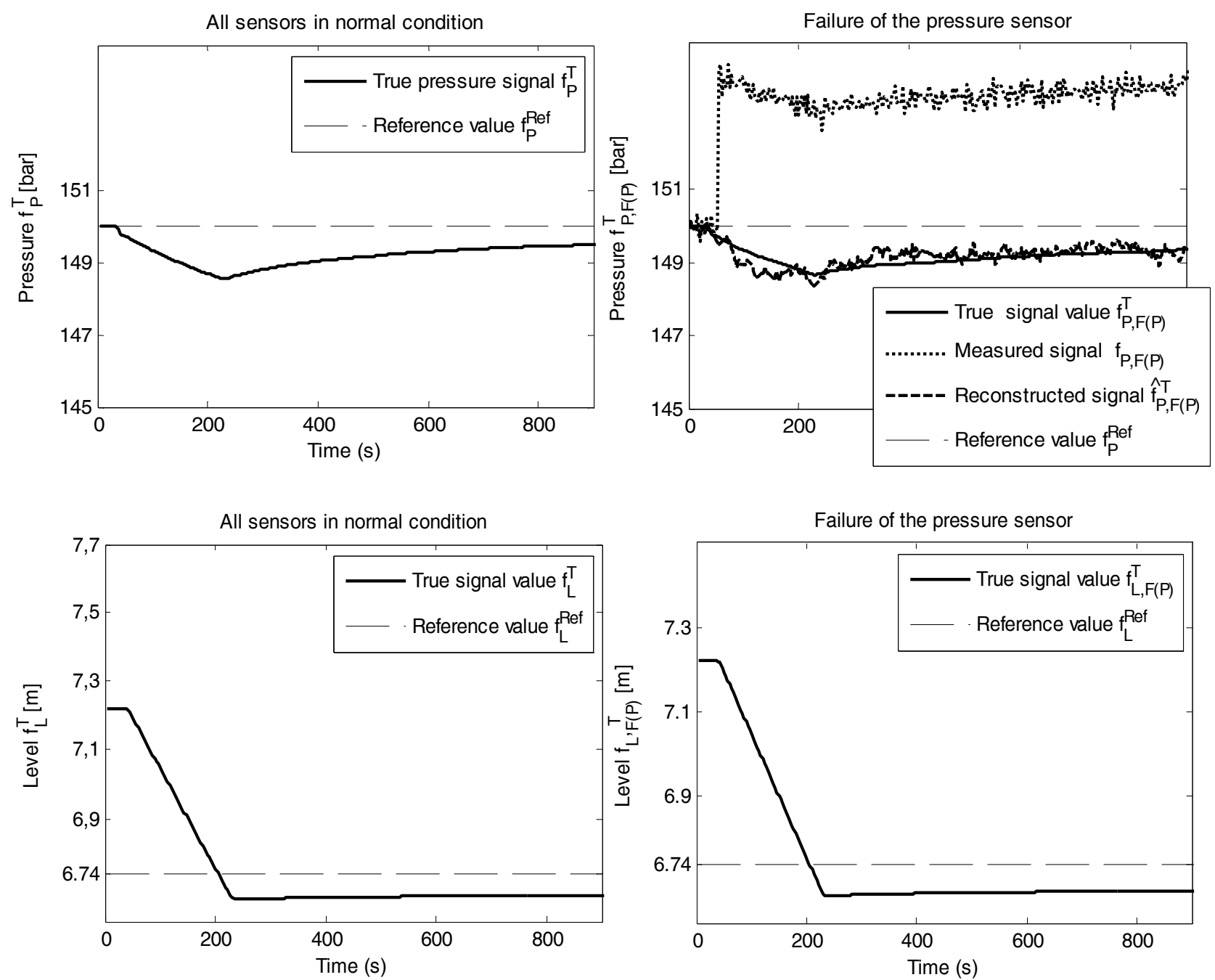

Fig. 15: evolution of the pressure (upper) and of the level (bottom) in the pressurizer when all the sensors are in normal conditions (left), and when a constant bias fault of +4.5 bar on the pressure measurement is simulated and the measured signal $\hat{f}_{i_{I}}^{T}$ is used as input to the controller (right). 

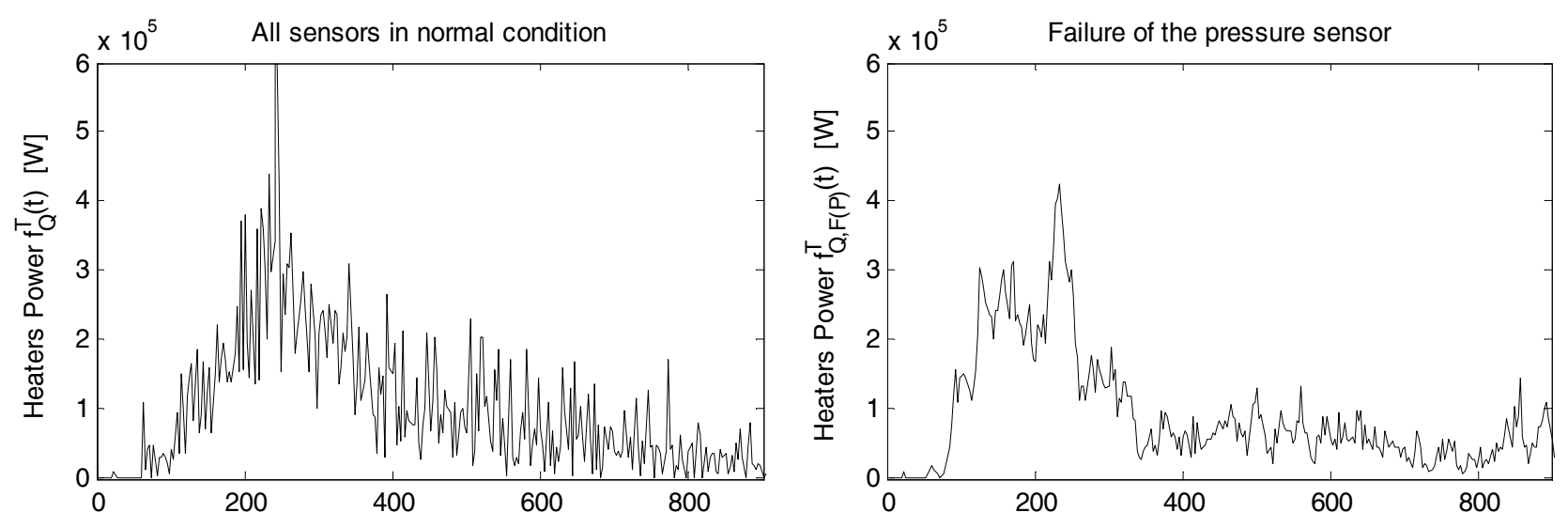

Fig. 16: evolution of the sprayers mass flow rate (upper) and of the heaters (bottom) in the pressurizer when all the sensors are in normal conditions (left), and when a constant bias fault of +4.5 bar on the pressure measurement is simulated and the measured signal $\hat{f}_{i_{I}}^{T}$ is used as input to the controller (right).

\subsubsection{Comparison of the results obtained with or without fault detection, identification and signal reconstruction}

Assuming as reference the behaviour of the controller when all sensors are in normal conditions, the consequences of a fault to sensor $i_{I}$ with successive fault detection, identification and signal reconstruction are compared to those without. The deviation $d_{i_{C}}(t)=\left|f_{i_{C}}^{T}(t)-f_{i_{C}, F\left(i_{I}\right)}^{T}(t)\right|$ of the true value of the $i_{C}$-th controlled signal in case of normal sensor conditions $f_{i_{C}}^{T}$ and in case of fault $f_{i_{C}, F\left(i_{I}\right)}^{T}$ is considered. In particular two performance indicators are evaluated, providing local (eq.(7)) and integral (eq.(8)) information about the effects of the fault on the controlled system, respectively (the lower the indicators, the better the controller performance):

$$
\begin{gathered}
M d_{i_{C}}=\max _{t}\left[d_{i_{C}}(t)\right] \\
I d_{i_{C}}=\sqrt{\frac{1}{\left(t_{f}-t_{0}\right)} \int_{t_{0}}^{t_{f}}\left[d_{i_{C}}(t)\right]^{2} d t}
\end{gathered}
$$

Table 5 reports the performance indicators for the transient with a fault of the pressure sensor of Table 4, in the case with or without fault detection, identification and signal reconstruction.

Table 5

Comparison of the performance indicators values in case the fault detection, identification and reconstruction is applied or not.

\begin{tabular}{ccc}
\multicolumn{2}{c}{ not. } \\
NO FAULT DETECTION, \\
$\begin{array}{c}\text { IDENTIFICATION AND SIGNAL } \\
\text { RECONSTRUCTION }\end{array}$ & $\begin{array}{c}\text { FAULT DETECTION, } \\
\text { IDENTIFICATION AND SIGNAL } \\
\text { RECONSTRUCTION }\end{array}$ \\
\hline \hline$M d_{P}$ & 4.433 bar & $0.161 \mathrm{bar}$
\end{tabular}




\begin{tabular}{lcc}
$M d_{L}$ & $0.781 \mathrm{~m}$ & $0.002 \mathrm{~m}$ \\
$I d_{P}$ & $2.04 \cdot 10^{3} \mathrm{bar}$ & $0.05 \cdot 10^{3} \mathrm{bar}$ \\
$I d_{L}$ & $3.71 \cdot 10^{2} \mathrm{~m}$ & $0.007 \mathrm{~m}$ \\
\hline
\end{tabular}

As expected, the performance indicators are significantly lower (better) in the case in which fault detection, identification and signal reconstruction is applied, thus indicating that the objectives of the control system are better satisfied, i.e. the controlled variables values are kept closer to their setpoints.

To confirm these results a number of other system faults have been analyzed (Table 6 and Table 7 for the faults of the pressure and level sensors, respectively). To verify the controller performance under different operative conditions of the pressurizer, eight transients have been considered, keeping the surge line mass flow rate at the constant value $f_{\text {surge }}^{T}=+6 \mathrm{~kg} / \mathrm{s}$ (in-surge) or $f_{\text {surge }}^{T}=-6 \mathrm{~kg} / \mathrm{s}$ (out-surge) for different time durations. For each transient, the total mass of liquid entering or exiting the pressurizer, i.e., the integral of the surge line mass flow rate over the entire duration of the transient, reflects a variation of the PHT water temperature ranging from -4 to $+4{ }^{\circ} \mathrm{C}$.

Table 6

Pressurizer condition during the simulated faulty transients.

\begin{tabular}{cc}
\hline FAulty SENSOR & Pressure sensor \\
TyPe OF Fault & Constant bias with 16 different fault magnitude values \\
FAult Magnitude & $M_{B} \in[ \pm 0.45 \pm 0.75 \pm 1.0 \pm 1.5 \pm 3.0 \pm 4.5 \pm 6.0 \pm 9.0]$ bar \\
BEgINNING TIME & $t=50 \mathrm{~s}$ \\
\hline
\end{tabular}

Table 7

Pressurizer condition during the simulated faulty transients.

\begin{tabular}{cc}
\hline & Pressurizer condition during the simulated faulty transients. \\
\hline FAulty SENSOR & Level sensor \\
TyPE OF FAUlT & Constant bias with 16 different fault magnitude values \\
FAULT MAGNITUDE & $M_{B} \in[ \pm 0.10 \pm 0.20 \pm 0.35 \pm 0.50 \pm 0.75 \pm 1.0 \pm 1.2 \pm 1.5] \mathrm{m}$ \\
BEGINNING TIME & $t=50 \mathrm{~s}$ \\
\hline
\end{tabular}

In particular, two tests have been performed by considering:

1) the 16 sensor faults of Table 6 and Table 7 occur during each of the eight pressurizer transients;

2) as in 1) but applying fault detection, identification and signal reconstruction.

To evaluate the performance in test 2, the delay time $\bar{\tau}$ necessary to detect the fault after its occurrence is considered. Fig. 17 shows the mean value of the delay times obtained in the eight transients simulated for each fault magnitude, in case of pressure (left) or level (right) fault. 

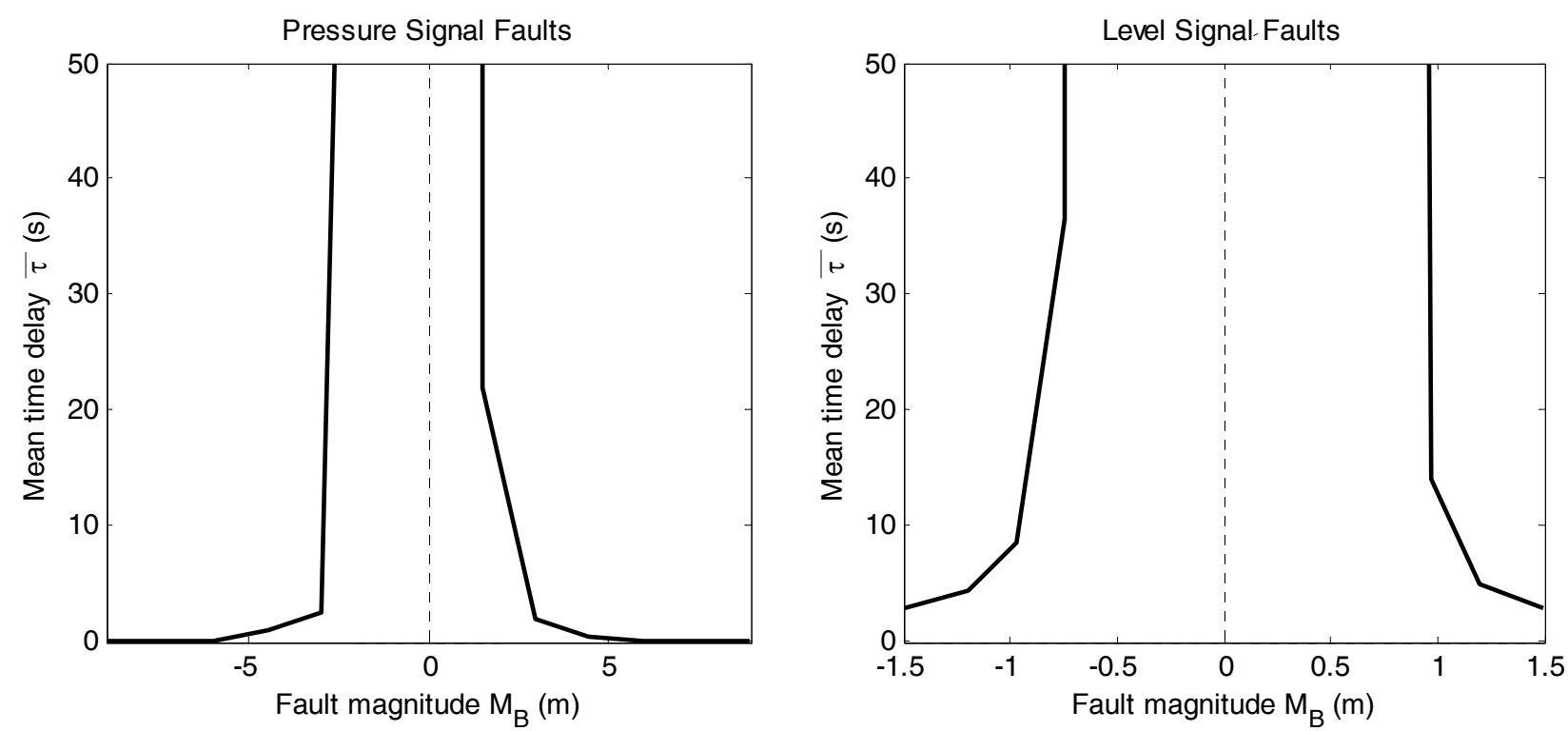

Fig. 17: mean fault detection time delay vs fault magnitude.

One can notice that as the fault magnitude $M_{B}$ gets smaller, the detection time $\bar{\tau}$ increases until the fault is too small to be detected; in particular, faults to the pressure sensor with an absolute value of their magnitude lower than $\left|M_{B}\right|=1.50$ bar are not detected; similarly, faults of the level sensor with an absolute value of their magnitude lower than $\left|M_{B}\right|=0.75 \mathrm{~m}$ are not detected. The numerical values of the mean time delays $\bar{\tau}$ before detecting the faults are reported in Table 8 .

Table 8

Mean fault detection time delay $\bar{\tau}$ for the smallest detectable faults.

\begin{tabular}{cc}
\hline FAULT $M_{B}$ & TIME DELAY $\bar{\tau}$ \\
\hline \hline$-1.5 \mathrm{bar}$ & $192 \mathrm{~s}$ \\
$+1.5 \mathrm{bar}$ & $22 \mathrm{~s}$ \\
$-0.75 \mathrm{~m}$ & $37 \mathrm{~s}$ \\
$+0.75 \mathrm{~m}$ & $591 \mathrm{~s}$ \\
\hline
\end{tabular}

In tests 1 and 2, for each values of the fault magnitude $M_{B}$ affecting signal $f_{i_{I}}$, the performance indicator $M d_{i_{C}}$ is computed as the maximum of $d_{i_{C}}(t)$ over the eight simulated transients, whereas a mean integral cost indicator $\overline{I d}_{i_{C}}$ is obtained by averaging the eight performance indicators $I d_{i_{C}}$ obtained for each transient.

Fig. 18 shows the values of the performance indicators $M d_{P}$ (upper left), $M d_{L}$ (upper right), $\overline{I d}_{P}$ (bottom left) and $\overline{I d}_{L}$ (bottom right) in correspondence of different values of the pressure sensor fault magnitude $M_{B}$ in the case in which fault detection, identification and signal reconstruction is applied (test 2, continuous line) or not (test 1, dotted line); notice that the maximum (worst) values of the performance indicators in test 2 are obtained in case of faults of small magnitude due to the fact that these faults are not 
detected or are detected with a significant time delay $\tau$ : thus, the signal reconstruction module is not working for the entire duration of the fault or for its first $\tau$ time instants.

Similar results are shown in Fig. 20 in the case of level sensor faults. Since the tolerated error band $B_{L}^{\varepsilon}=2$ $\mathrm{m}$ is quite large, the faults of magnitude included in the interval $M_{B}=[-1.2+1.0] \mathrm{m}$ have no consequences on the correct control of the process.

These results confirm that the sensor diagnostic procedure integrated in the controller of the PWR pressurizer allows improving significantly the control of the process not only in some critical local points as stated by the $M d_{i_{C}}$ indicators, but also during the entire transient as demonstrated by the $\overline{I d}_{i_{C}}$ integral indicators. 

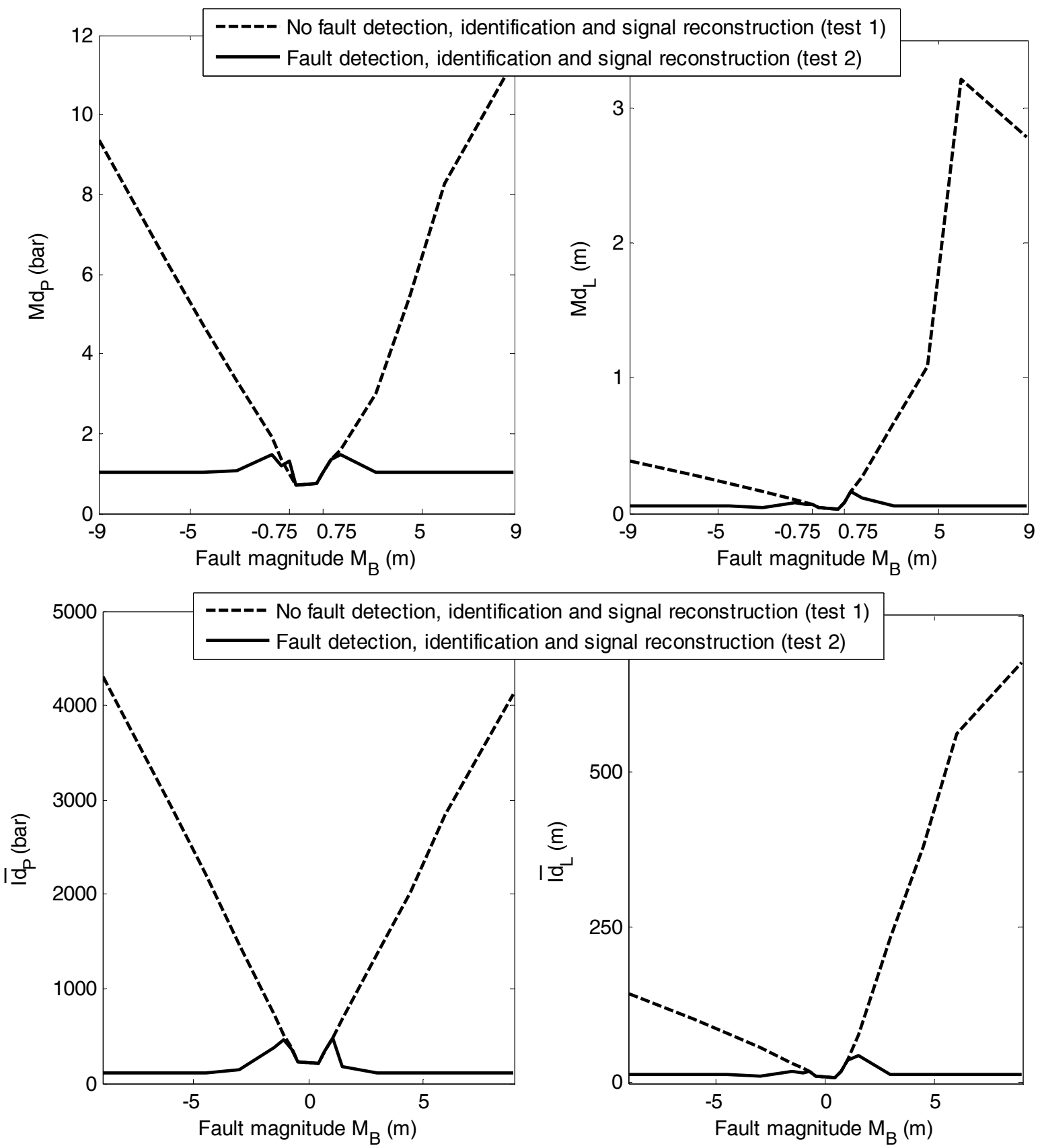

Fig. 18: $M d_{P}$ (upper left), $M d_{L}$ (upper right), $\overline{I d}_{P}$ (bottom left) and $\overline{I d}_{L}$ (bottom right) indicators versus the magnitude $M_{B}$ of the constant bias fault added to the pressure measurement. 

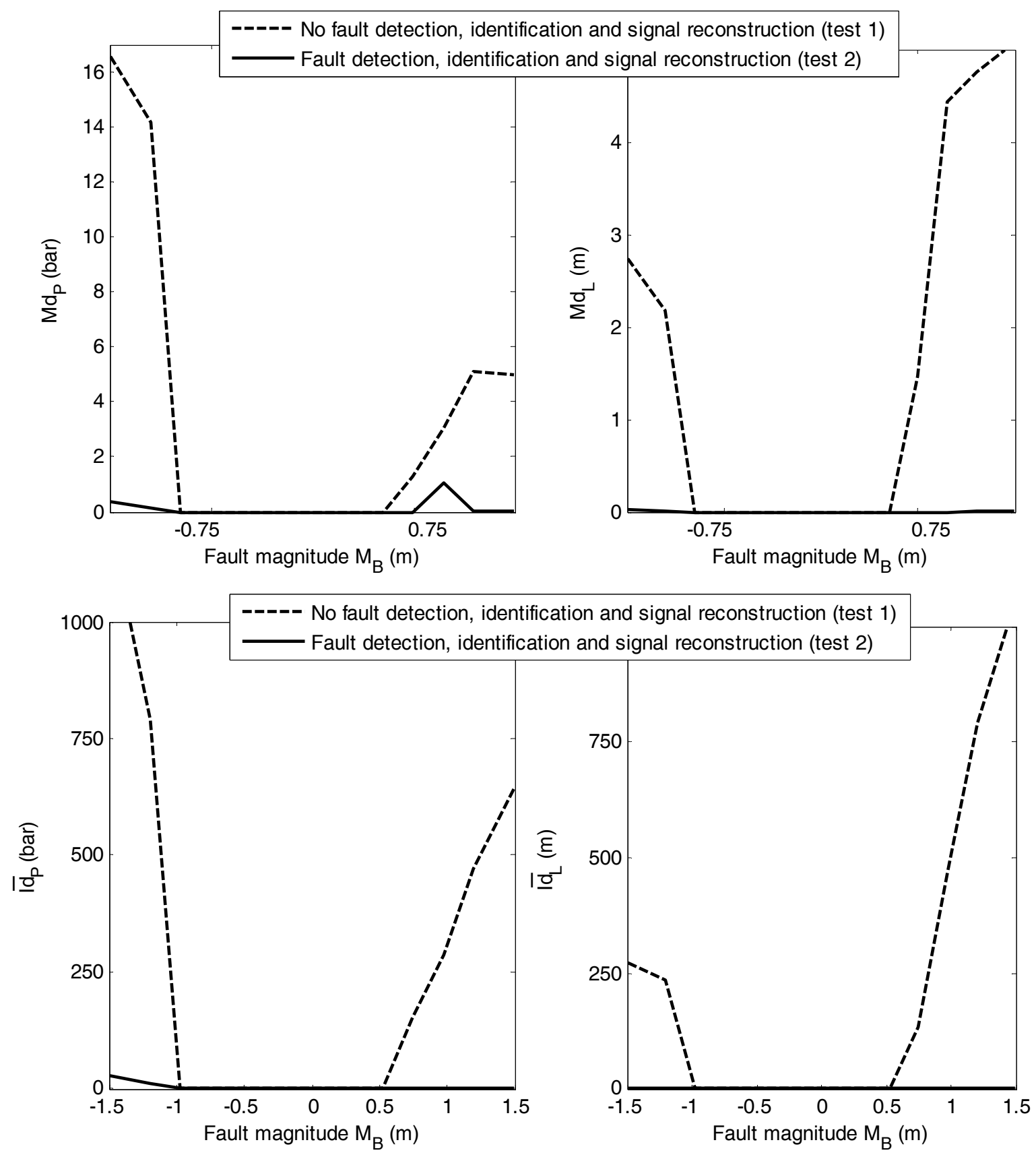

Fig. 19: $M d_{P}$ (upper left), $M d_{L}$ (upper right), $\overline{I d}_{P}$ (bottom left) and $\overline{I d}_{L}$ (bottom right) indicators versus the magnitude $M_{B}$ of the constant bias fault added to the level measurement.

\subsection{Results discussion}

The results presented in this Section demonstrate that with the fault detection, identification and signal reconstruction approach proposed it is possible to effectively control the pressurizer when a fault occurs on the pressure or level sensors, in the sense that the controlled variables obtained using the reconstructed signals do not diverge from their setpoints and the system behaviour is kept similar to the one under normal, fault-free sensors operation. 
Faults of different magnitudes within a given range have been simulated to verify these results; larger faults magnitudes would not have been realistic. Only the results obtained for the constant bias fault type have been presented; nevertheless, similar results have been achieved also in the case of noise-type faults.

The entire fault detection, identification and reconstruction procedure requires about $0.3 \mathrm{~s}$ for the elaboration of each measurement performed with a standard personal computer; the delay thus introduced in the controller decision is compatible with the typical time constant of a pressurizer and does not affect the effective control of the system.

The constant values of $+6 \mathrm{~kg} / \mathrm{s}$ in case of in-surge or $-6 \mathrm{~kg} / \mathrm{s}$ for the out-surge assigned to the surge line mass flow rate have not been chosen too high in order to avoid falling in a region not represented by the training patterns used to train the model; if the surge line mass flow rate and at the same time the total mass of liquid entering the pressurizer have high values, the pressure and level can take too high (in-surge) or too low (outsurge) values, which are not represented by any pattern of the training set. In this case, the measurements cannot be reconstructed properly by the model which would be extrapolating its reconstructions.

Another case in which the model looses its validity is when the charging/letdown mass flows rates are different from zero. During standard transients the controller does not need to act on the charging/letdown flows; as a consequence, all training patterns have charging/letdown mass flow rates equal to zero and thus the signal has not been used to build the model. In some particular cases, for example in case of level sensor faults, a controller action on the charging/letdown flows might take place; this situation is not handled by the current set up of the approach.

In all these cases, the diagnostic system should signal that the measurements are out of the range of validity of the model and that the methodology should not be applied.

For lower values of the surge line mass flow rate, it has been verified that the consequences of faults are smaller and at the same time the proposed approach work best because the measured signals fall in the region with the highest density of training patterns, where the signal validation model is more accurate.

\section{Conclusions}

An approach for the detection and identification of faulty sensors and the reconstruction of their erroneous measurements has been proposed. The effectiveness of the approach in increasing the performance of system control when a fault affects the controller input signals is confirmed by the results obtained from its application to the control of a PWR pressurizer.

The reconstruction provided by the signal validation and reconstruction model described in Section 3 provides good estimates of the true values of the measured variables and can be used for control.

The boundaries of operation of the proposed approach are defined by the region in which the training patterns fall; techniques exist to warn when the pattern under reconstruction is outside such region and is thus, no longer reliable (Fantoni et al., 1997). 
The case of simultaneous presence of multiple faults has not been considered: future work should test the approach in this situation. Also, the problem of fault propagation has not been tackled directly: the definition of a refined strategy for the distinction of false alarms will be considered in future research.

\section{Acknowledgments}

The authors are pleased to thank G. Gola, M. Hoffmann and D. Roverso of the Institute for Energy Technology (IFE), OECD Halden Reactor Project, Norway for supplying the tool implementing the SPRT.

\section{Appendix A}

\section{Acronyms}

$\mathrm{k}-\mathrm{nn}=\mathrm{k}$-nearest neighbors

$\mathrm{LF}=$ Local Fusion

PCA = Principal Components Analysis

$\mathrm{PID}=$ Proportional Integral Derivative

PHT $=$ Primary Heat Transfert

PWR $=$ Pressurized Water Reactor

RFSE $=$ Random Feature Selection Ensemble

SPRT $=$ Sequential Probability Ratio Test

\section{Nomenclature}

Signals:

$n=$ Total number of sensors' signals available

$n_{C}=$ Total number of controlled signal

$n_{I}=$ Total number of controller input signal

$f_{i}=$ Sensor measurement

$\hat{f}_{i}=$ Estimate of the $i$-th sensor measurement provided by a model

$\hat{f}_{i}^{j}=$ Estimate of the $i$-th sensor measurement provided by a model at the $j$-th iteration of the reconstruction

$f_{i}^{T}=$ True value of the physical quantity measured by the $i$-th sensor

$\hat{f}_{i}^{T}=$ Final reconstruction of $f_{i}^{T}$

$f_{i_{C}, F\left(i_{I}\right)}^{T}=$ True value of the $i_{C}$-th controlled signal in case of fault of the $i_{I}$-th controller input signal

Signal Validation:

$\mathbf{X}=$ Data set

$\mathbf{X}_{T R N}=$ Training data subset

$\mathbf{X}_{T S T}=$ Test data subset

$N=$ Total number of signal patterns

$N_{\text {TRN }}=$ Number of training patterns

$N_{\text {TST }}=$ Number of test patterns

$\mathbf{F}_{h}=$ Subset of signals

$m=$ Number of signals in each subsets $\mathbf{F}_{h}$

$H=$ Total number of models

$H_{i}=$ Number of models reconstructing the $i$-th signal

$\hat{f}_{i}^{h}=$ Reconstruction made by the $h$-th model of the $i$-th signal 
$Q_{T R N}=$ Subset of training patterns similar to the test pattern, also called neighborhood of the test pattern

$k=$ Cardinality of the neighborhood $Q_{T R N}$

$m a e_{i, Q_{T R N}}^{h}=$ Mean absolute error of the $h$-th model in the reconstruction of the $i$-th signal of the patterns in the set $Q_{T R N}$

$m e_{i, Q_{T R N}}^{h}=$ Mean error of the $h$-th model in the reconstruction of the $i$-th signal of the patterns in the set $Q_{T R N}$

$b_{i}^{h}=$ Bias correction associated to the $h$-th model for the reconstruction of the $i$-th signal

$w_{i}^{h}=$ Weight associated to the $h$-th model for the reconstruction of the $i$-th signal

Fault detection:

$H p_{i}^{0}=$ Hypothesis of $i$-th sensor being in normal condition

$H p_{i}^{1}=$ Hypothesis of $i$-th sensor being in faulty condition

$e_{i}(t)=f_{i}(t)-\hat{f}_{i}^{l}(t)=$ Residual signal

$m_{i}^{j}=$ Mean value of the residual $e_{i}$ under the hypothesis $H p_{i}^{j}, j=0,1$

$\sigma_{i}^{j}=$ Standard deviation of the residual $e_{i}$ under the hypothesis $H p_{i}^{j}, j=0,1$

$\alpha=$ False alarm probability

$\beta=$ Missed alarm probability

$\left.B_{U}=\log [(1-\beta) / \alpha)\right]=$ Upper thresholds for $\lambda_{i}(t)$

$B_{L}=\log [\beta /(1-\alpha)]=$ Lower thresholds for $\lambda_{i}(t)$

$\lambda_{i}(t)=\log$ likelihood ratio of the residual $e_{i}$

$\tau=$ Delay time necessary for fault detection

Control:

$\varepsilon_{i_{C}}=f_{i_{C}}^{R e f}-f_{i_{C}}=$ Distance between the measured value of the $i_{C}$-th controlled signal and its reference value

$B_{i}^{\varepsilon}=$ Band of tolerated error $\varepsilon_{i}$

$d_{i_{C}}=$ deviation $\left|f_{i_{C}}^{T}(t)-f_{i_{C}, F\left(i_{I}\right)}^{T}(t)\right|$ of the true $i_{C}$-th signal in case of sensor fault and in case of normal sensor conditions.

$M d_{i_{C}}=\max _{t}\left[d_{i_{C}}(t)\right]=$ Local performance indicator computed for the $i_{C}$-th signal

$I d_{i_{C}}=\sqrt{\frac{1}{\left(t_{f}-t_{0}\right)} \int_{t_{0}}^{t_{f}}\left[d_{i_{C}}(t)\right]^{2} d t}=$ Integral performance indicator computed for the $i_{C}$-th signal

PWR pressurizer model:

$P=$ Pressure

$h_{L}=$ Enthalpy of the liquid in the pressurizer

$h_{V}=$ Enthalpy of the vapour in the pressurizer

$h_{\text {surge }}=$ Enthalpy of the surge line liquid water

$h_{c l}=$ Enthalpy of the charging/letdown liquid water

$h_{s p}=$ Enthalpy of the sprayers liquid water

$h_{F}(P)=$ Enthalpy of the liquid in saturation condition at pressure $P$

$h_{G}(P)=$ Enthalpy of the vapour in saturation condition at pressure $P$

$T_{L}\left(P, h_{L}\right)=$ Temperature of the liquid

$T_{V}\left(P, h_{V}\right)=$ Temperature of the vapour

$T_{F}(P)=$ Temperature of the liquid in saturation condition at pressure $P$ 
$T_{G}(P)=T_{F}=$ Temperature of the vapour in saturation condition at pressure $P$

$V=$ Volume of the pressurizer

$V_{V}=$ Volume of the vapour in the pressurizer

$V_{L}=V-V_{V}=$ Volume of the liquid in the pressurizer

$S=$ Surface of the pressurizer horizontal section

$L=V_{L} / S=$ Level of the liquid water in the pressurizer

$\dot{Q}=$ Heaters power

$\rho_{L}\left(P, h_{L}\right)=$ Density of the liquid water in the pressurizer

$\rho_{V}\left(P, h_{V}\right)=$ Density of the vapour in the pressurizer

$\rho_{F}\left(P, h_{F}\right)=$ Density of the liquid water in saturated conditions at pressure $P$

$\rho_{G}\left(P, h_{G}\right)=$ Density of the vapour in saturated conditions at pressure $P$

$\dot{m}_{v l v}=$ Relief valve mass flow rate

$\dot{m}_{\text {surge }}=$ Surge line mass flow rate

$\dot{m}_{s p}=$ Sprayers mass flow rate

$\dot{m}_{c s}=\dot{m}_{s p} \frac{\left(h_{F}-h_{s p}\right)}{\left(h_{G}-h_{F}\right)}=$ Mass flow rate of the steam condensed by the sprayers

$\dot{m}_{f l}=$ Liquid flashes mass flow rate

$\dot{m}_{r o}=$ Condensing steam mass flow rate

$\dot{m}_{c l}=$ Charging/letdown mass flow rate

$\alpha_{h}=$ Heat transfer coefficient

$P^{0}=$ Pressure initial condition

$V_{V}^{0}=$ Vapour volume initial condition

$T_{\text {surge }}^{0}=$ Initial condition of the surge line water temperature

$T_{c l}^{0}=$ Initial condition of the charging/letdown water temperature

$T_{s p}^{0}=$ Initial condition of the sprayers' water temperature

$h_{\text {surge }}^{0}\left(P^{0}, T_{\text {surge }}^{0}\right)=$ Initial condition of the surge line water enthalpy

$h_{c l}^{0}\left(P^{0}, T_{c l}^{0}\right)=$ Initial condition of the charging/letdown water enthalpy

$h_{s p}^{0}\left(P^{0}, T_{s p}^{0}\right)=$ Initial condition of the sprayers' water enthalpy

$T_{L}^{0}=$ Initial condition of the pressurizer liquid water temperature

$T_{V}^{0}=$ Initial condition of the vapour temperature

$h_{L}^{0}\left(P^{0}, T_{L}^{0}\right)=$ Initial condition of the pressurizer liquid water enthalpy

$h_{V}^{0}\left(P^{0}, T_{V}^{0}\right)=$ Initial condition of the vapour enthalpy

\section{Appendix B}

\section{Pressurizer model equations:}

The pressurizer model is designed to handle the four possible situations listed in Table B.1.

Table B.1

Liquid water and vapour conditions in the four situations tackled by the SIMULINK pressurizer model.

\begin{tabular}{ccc}
\hline CASE & $\begin{array}{c}\text { LIQUID WATER } \\
\text { CONDITION }\end{array}$ & $\begin{array}{c}\text { VAPOUR } \\
\text { CONDITION }\end{array}$ \\
\hline \hline $\mathrm{A}$ & Subcooled & Overheated
\end{tabular}




\begin{tabular}{ccc} 
B & Saturated & Overheated \\
C & Subcooled & Saturated \\
D & Saturated & Saturated \\
\hline
\end{tabular}

Each situation is represented by a different dynamic model of the pressurizer described by the system of differential equations $\boldsymbol{\Psi} \cdot \dot{z}=\eta$, where $\boldsymbol{\Psi}$ is the coefficients matrix, $\eta$ is the inputs vector and $z$ is the state variables vector.

The elements of $\boldsymbol{\Psi}, \eta$ and $z$ are here detailed for each situation considered.

Case $A . \quad h_{L}<h_{F}$ and $h_{V}>h_{G}$

$\boldsymbol{\Psi}=\left[\begin{array}{cccc}V_{V} \frac{\partial \rho_{V}}{\partial P} & \rho_{V} & V_{V} \frac{\partial \rho_{V}}{\partial h_{V}} & 0 \\ \left(V-V_{V}\right) \frac{\partial \rho_{L}}{\partial P} & -\rho_{L} & 0 & \left(V-V_{V}\right) \frac{\partial \rho_{L}}{\partial h_{L}} \\ -V_{V} & 0 & V_{V} \rho_{V} & 0 \\ -\left(V-V_{V}\right) & 0 & 0 & \left(V-V_{V}\right) \rho_{L}\end{array}\right], \quad z=\left[\begin{array}{c}P \\ V_{V} \\ h_{V} \\ h_{L}\end{array}\right]$

$\eta=\left[\begin{array}{c}-\dot{m}_{v l v}-\dot{m}_{c s} \\ \dot{m}_{c s}+\dot{m}_{\text {surge }}+\dot{m}_{s p}+\dot{m}_{c l} \\ \dot{m}_{c s}\left(h_{V}-h_{G}\right)-\alpha_{h} S\left(T_{V}-T_{L}\right) \\ \dot{m}_{\text {surge }}\left(h_{\text {surge }}-h_{L}\right)+\dot{m}_{s p}\left(h_{s p}-h_{L}\right)+\dot{m}_{c l}\left(h_{c l}-h_{L}\right)+\dot{m}_{c s}\left(h_{G}-h_{L}\right)+\dot{Q}+\alpha_{h} S\left(T_{V}-T_{L}\right)\end{array}\right]$

Case B. $\quad h_{L}=h_{F}$ and $h_{V}>h_{G}$

$\begin{aligned} \boldsymbol{\Psi} & =\left[\begin{array}{cccc}V_{V} \frac{\partial \rho_{V}}{\partial P} & \rho_{V} & V_{V} \frac{\partial \rho_{V}}{\partial h_{V}} & -1 \\ \left(V-V_{V}\right) \frac{d \rho_{F}}{d P} & -\rho_{F} & 0 & 1 \\ -V_{V} & 0 & V_{V} \rho_{V} & \left(h_{V}-h_{G}\right) \\ \left(V-V_{V}\right)\left(\rho_{F} \frac{d h_{F}}{d P}-1\right) & 0 & 0 & \left(h_{G}-h_{F}\right)\end{array}\right], \quad z=\left[\begin{array}{c}P \\ V_{V} \\ h_{V} \\ m_{f l}\end{array}\right], \\ \eta & \left.\begin{array}{c}\dot{m}_{c s}+\dot{m}_{\text {surge }}+\dot{m}_{s p}+\dot{m}_{c l} \\ \dot{m}_{c s}\left(h_{V}-h_{G}\right)-\alpha_{h} S\left(T_{V}-T_{F}\right) \\ \dot{m}_{\text {surge }}\left(h_{\text {surge }}-h_{F}\right)+\dot{m}_{s p}\left(h_{s p}-h_{F}\right)+\dot{m}_{c l}\left(h_{c l}-h_{F}\right)+\dot{m}_{c s}\left(h_{G}-h_{F}\right)+\dot{Q}+\alpha_{h} S\left(T_{V}-T_{F}\right)\end{array}\right]\end{aligned}$

Case C. $\quad h_{L}<h_{F}$ and $h_{V}=h_{G}$

$\boldsymbol{\Psi}=\left[\begin{array}{cccc}V_{V} \frac{d \rho_{G}}{d P} & \rho_{G} & 1 & 0 \\ \left(V-V_{V}\right) \frac{\partial \rho_{L}}{\partial P} & -\rho_{L} & -1 & \left(V-V_{V}\right) \frac{\partial \rho_{L}}{\partial h_{L}} \\ V_{V}\left(\rho_{G} \frac{d h_{G}}{d P}-1\right) & 0 & \left(h_{F}-h_{G}\right) & 0 \\ -\left(V-V_{V}\right) & 0 & \left(h_{L}-h_{F}\right) & \left(V-V_{V}\right) \rho_{L}\end{array}\right], \quad z=\left[\begin{array}{c}P \\ V_{V} \\ m_{r o} \\ h_{L}\end{array}\right]$ 
$\eta=\left[\begin{array}{c}-\dot{m}_{v l v}-\dot{m}_{c s} \\ \dot{m}_{c s}+\dot{m}_{\text {surge }}+\dot{m}_{s p}+\dot{m}_{c l} \\ -\alpha_{h} S\left(T_{G}-T_{L}\right) \\ \dot{m}_{\text {surge }}\left(h_{\text {surge }}-h_{L}\right)+\dot{m}_{s p}\left(h_{s p}-h_{L}\right)+\dot{m}_{c l}\left(h_{c l}-h_{L}\right)+\dot{m}_{c s}\left(h_{G}-h_{L}\right)+\dot{Q}+\alpha_{h} S\left(T_{G}-T_{L}\right)\end{array}\right]$

Case D. $\quad h_{L}=h_{F}$ and $h_{V}=h_{G}$

$\boldsymbol{\Psi}=\left[\begin{array}{cccc}V_{V} \frac{d \rho_{G}}{d P} & \rho_{G} & 1 & -1 \\ \left(V-V_{V}\right) \frac{d \rho_{L}}{d P} & -\rho_{F} & -1 & 1 \\ V_{V}\left(\rho_{G} \frac{d h_{G}}{d P}-1\right) & 0 & \left(h_{F}-h_{G}\right) & 0 \\ -\left(V-V_{V}\right)\left(\rho_{F} \frac{d h_{F}}{d P}-1\right) & 0 & 0 & \left(h_{G}-h_{F}\right)\end{array}\right], \quad z=\left[\begin{array}{c}P \\ V_{V} \\ m_{r o} \\ m_{f l}\end{array}\right]$,

$\eta=\left[\begin{array}{c}-\dot{m}_{v l v}-\dot{m}_{c s} \\ \dot{m}_{c s}+\dot{m}_{\text {surge }}+\dot{m}_{s p}+\dot{m}_{c l} \\ 0 \\ \dot{m}_{\text {surge }}\left(h_{\text {surge }}-h_{F}\right)+\dot{m}_{s p}\left(h_{s p}-h_{F}\right)+\dot{m}_{c l}\left(h_{c l}-h_{F}\right)+\dot{m}_{c s}\left(h_{G}-h_{F}\right)+\dot{Q}\end{array}\right]$

Notice that the elements of the coefficients' matrix $\Psi$ depend from the state variables so that the model is nonlinear. In practice at each time step the SIMULINK model checks in which one of the four possible cases the pressurizer is and evaluates the coefficients' matrix $\boldsymbol{\Psi}$ according to values of the state variables at the previous time step.

\section{Appendix C}

\section{Pressurizer control scheme:}

The main function of a pressurizer is that of maintaining the pressure in the primary system of the plant at the reference value (150 bar) to give adequate overpressure, suppress pump cavitations and avoid bulk boiling. The pressurizer is also designed to accommodate normal volume surges. The level $L$ of the liquid water contained in it must also be controlled in order to avoid emptying or overfilling its volume.

In order to allow this component to fulfil its functions, it is necessary to continuously control that the value of the water level $L$ and the pressure $P$ in the pressurizer agree with the desired reference value $f_{L}^{\text {Ref }}$ and $f_{P}^{\text {Ref }}$. Fig. C.1 shows how decisions are generated by the controller. 


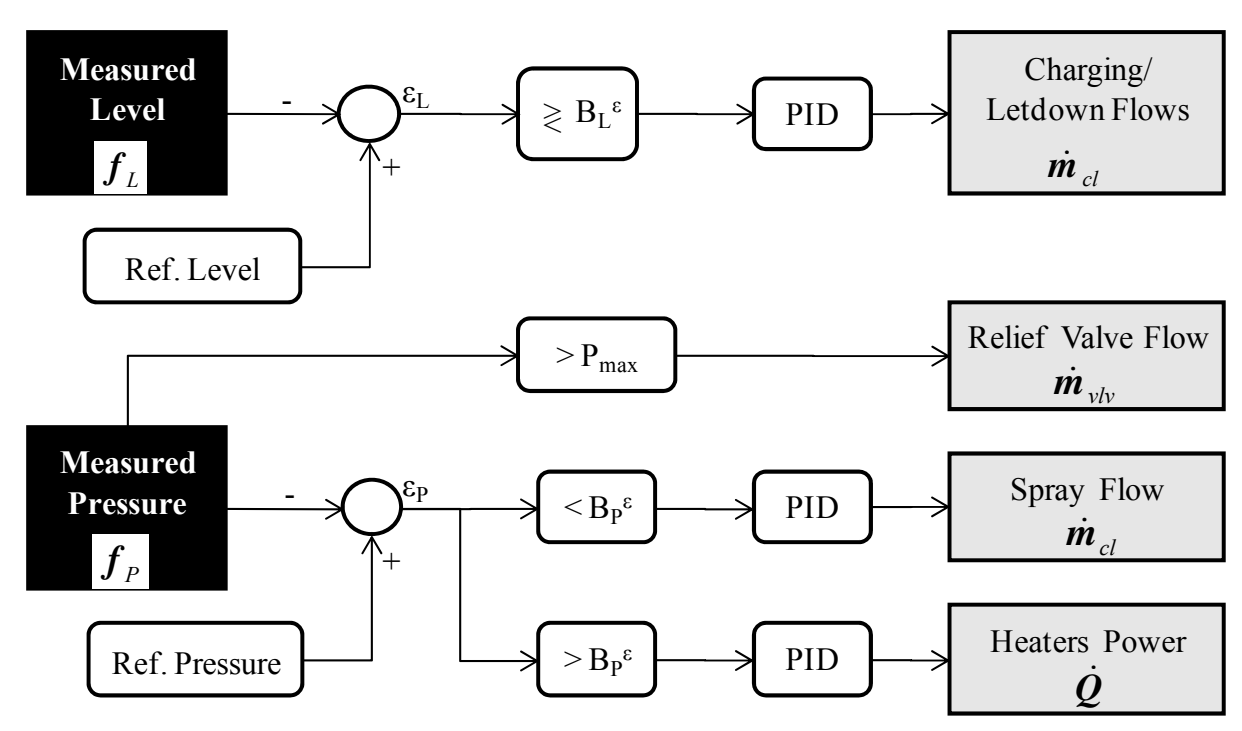

Fig C.1: pressurizer control scheme

The pressure measurement $f_{P}$ is compared with the constant pressure setpoint of 150 bar, whereas the measured value of the level $f_{L}$ is compared with a reference value which varies according with the variation of the temperature in the PHT system. The controller intervenes only if the controlled signal $i_{C}$, which is at the same time a controller input signal, differs from its reference value $f_{i_{C}}^{R e f}$ of a quantity greater than the tolerated error band, here set to the values $B_{P}^{\varepsilon}=0.2$ bar for the pressure and $B_{L}^{\varepsilon}=2 \mathrm{~m}$ for the level. Notice that a stricter control condition is applied to the pressure than to the level. When one of the tolerated error band is exceeded, the difference $\varepsilon_{i_{C}}=f_{i_{C}}^{R e f}-f_{i_{C}}$ between the measured signal and its reference value is given in input to a proportional-integral-derivative (PID) controller which generates the control signal for the corresponding actuator, i.e., the charging/letdown flows, the sprayers or the heaters. The relief valve, instead, is a safety device which opens only when the pressure exceeds a limit value (here set equal to $P^{\max }=165$ bar) and has a constant mass flow rate.

In case of low pressure, the heaters are turned on. For a more precise control of the supplied power only one out of five heaters can deliver variable PID controlled power, whereas the other four units are back-up heaters run by control relays. All units have a maximum power of $320 \mathrm{~kW}$ each. The pressure is increased as a consequence of the greater mass flow rate $\dot{m}_{f l}$ generated by liquid water flashes produced by warming up the liquid water. If the pressure is getting too high the control signal generated by the corresponding PID is sent to the sprayers which start injecting nebulised water into the pressurizer with a maximum mass flow rate of $7 \mathrm{~kg} / \mathrm{s}$. The flow of nebulised cold water coming from the sprayers causes the condensation of the steam, thus inducing a pressure decrease. In a similar way, the deviations of the level from the reference value $f_{L}^{\text {Ref }}$ are controlled by regulating the charging/letdown mass flows rates.

\section{References}

Aly, M.A., Atiya, A.F., 2006. Novel methods for the feature subset ensembles approach, International Journal of Artificial Intelligence and Machine Learning, Vol. 6, No. 4. 
Baraldi, P., Zio, E., Gola, G., Roverso, D., Hoffmann, M., 2008. "Genetic algorithms for signal grouping in sensor validation: a comparison of the filter and wrapper approaches", Journal of Risk and Reliability (JRR), Proc. IMechE, Vol. 222, Part O, pp. 189-206.

Baraldi, P., Zio, E., Gola, G., Roverso, D., Hoffmann, M., 2009a. A procedure for the reconstruction of faulty signals by means of an ensemble of regression models based on principal components analysis, NPIC-HMIT 2009 Topical Meeting, April 5-9.

Bonissone, P.P., Xue, F., Subbu, R., 2008. Fast meta-models for local fusion of multiple predictive models, Appl. Soft Comput. J., doi:10.1016/j.asoc.2008.03.006.

Bryll, R., Gutierrez-Osuna, R., Quek, F., 2003. Attribute bagging: improving accuracy of classifiers ensembles by using random feature subsets, Pattern Recognition, Vol. 36, pp. 1291-1302.

Chenggang, Y., Bingjing, S., 2006. Eliminating false alarms caused by fault propagation in signal validation by subgrouping, Progress in Nuclear Energy, Volume 48, Issue 4, May, Pages 371-379

Chevalier, R., Provost, D., Seraoui, R., 2009. Assessment of statistical and classification models for monitoring EDF's assets, NPIC-HMIT 2009 Topical Meeting, April 5-9.

Diamantaras, K.I., Kung, S.Y., 1996. Principal component neural networks: theory and applications, John Wiley \& Sons, Inc. New York, NY, USA.

Fantoni, P.F., Mazzola, A., 1996. Multiple-Failure Signal Validation in Nuclear Power Plants using Artificial Neural Networks, Nuclear technology, Vol. 113, No. 3, pp. 368-374.

Fantoni, P.F., Figedy, S., Papin, B., 1997. A Neuro-Fuzzy Model Applied to Full Range Signal Validation of PWR Nuclear Power Plant Data, Second OECD Specialist Meeting on Operator Aids for Severe Accident Management (SAMOA-2), Lion, France, Sept. 8-10.

Fantoni, P.F., Hoffmann, M., Shankar, R., Davis, E.L., 2003. On-line Monitoring of Instrument Channel Performance in Nuclear Power Plant using PEANO, Progress in Nuclear Energy, Vol. 43, No. 1-4, Pages 83-89.

Gross, K.C., Kumenik, K.E., 1991. Sequential probability ratio test for nuclear power plant component surveillance, Nuclear Technology 93, pp. 131-137,

Hashemian, H.M., 2004. Sensor Performance and Reliability, ISA.

Hashemian, H.M., 2006. Maintenence of Process Instrumentation in Nuclear Power Plants, Springer.

Hoffmann, M., 2006. Signal Grouping Algorithm for an Improved On-line Calibration Monitoring System, Proceedings of FLINS, Genova, Italy, Aug. 2006.

Holbert, K.E., Upadhyaya, B.R., 1990. An Integrated Signal Validation for Nuclear Power Plants, Nuclear Technology, Vol. 92, No. 3, pp. 411-427.

Johnson, F., 2008. Nuclear Reactor Controls and Instrumentation, Wexford College Press.

Jolliffe, I.T., 2002. Principal Component Analysis, Springer Eds.

Kuridan, R.M., Beynon, T.D., 1998. A linearized non steady model for the pressurizer of the safe integral reactor concept, Progress in Nuclear Energy, Vol. 33.

Moore, B., 1981. Principal component analysis in linear systems: Controllability, observability, and model reduction, IEEE Transactions on Automatic Control, Vol. 26, Issue 1, Feb.

Roverso, D., Hoffmann, M., Zio, E., Baraldi, P., Gola, G., 2007. Solutions for plant-wide on-line calibration monitoring, Proc. ESREL 2007, Stavanger, Norway, Vol. 1, pp. 827-832.

Scholkopf, B., Smola, A., Muller, K.R., 1999. Kernel principal component analysis”, Advances in Kernel MethodsSupport Vector Learning.

Todreas, N., Kazimi, M.S., 1990. Nuclear Systems 1, Taylor \& Francis.

Wald, A., 1947. Sequential Analysis, New York: John Wiley \& Sons.

Yang, H.Y., Lee, S.H., Na, M.G., 2009. Monitoring and Uncertainty analysis of feedwater flow rate using data-based modeling method, IEEE Transactions on Nuclear Science, vol. 56, issue 4, Aug, pp. 2426-2433.

Zio, E., Baraldi, P., Gola, G., Roverso, D., Hoffmann, M., 2007. Genetic Algoritms for Grouping of Signals for System Monitoring and Diagnostics, Proc. ESREL 2007, Stavanger, Norway, Vol. 1, pp. 833-840. 